\title{
STICKY COUPLINGS OF MULTIDIMENSIONAL DIFFUSIONS WITH DIFFERENT DRIFTS
}

\author{
ANDREAS EBERLE AND RAPHAEL ZIMMER
}

\begin{abstract}
We present a novel approach of coupling two multidimensional and non-degenerate Itô processes $\left(X_{t}\right)$ and $\left(Y_{t}\right)$ which follow dynamics with different drifts. Our coupling is sticky in the sense that there is a stochastic process $\left(r_{t}\right)$, which solves a one-dimensional stochastic differential equation with a sticky boundary behavior at zero, such that almost surely $\left|X_{t}-Y_{t}\right| \leq r_{t}$ for all $t \geq 0$. The coupling is constructed as a weak limit of Markovian couplings. We provide explicit, non-asymptotic and long-time stable bounds for the probability of the event $\left\{X_{t}=Y_{t}\right\}$.
\end{abstract}

\section{INTRODUCTION}

Let $\left(B_{t}\right)$ and $\left(\tilde{B}_{t}\right)$ be $d$-dimensional Brownian motions. We consider two diffusion processes with values in $\mathbb{R}^{d}$ which follow dynamics with different drifts, i.e.

$$
\begin{aligned}
& d X_{t}=b\left(t, X_{t}\right) d t+d B_{t}, \quad X_{0}=x, \\
& d Y_{t}=\tilde{b}\left(t, Y_{t}\right) d t+d \tilde{B}_{t}, \quad Y_{0}=y .
\end{aligned}
$$

We assume that the drift coefficients $b, \tilde{b}: \mathbb{R}_{+} \times \mathbb{R}^{d} \rightarrow \mathbb{R}^{d}$ are locally Lipschitz. Moreover, we impose assumptions which imply that a geometric Lyapunov drift condition holds for (1) and that there is a constant $M>0$ such that uniformly $|b-\tilde{b}| \leq M$.

Diffusions with different drifts occur in many application areas. For example, one could consider a Langevin diffusion $\left(X_{t}\right)$ and a perturbation or approximation $\left(Y_{t}\right)$ of the latter. Other natural examples are McKean-Vlasov processes, where the drift coefficients depend not only on the current position of the process but also on the corresponding law. A natural question arising is how to obtain explicit bounds for the distance of $X_{t}$ and $Y_{t}$ in Kantorovich distances, e.g. in total variation norm. There are a few articles which try to answer this question in a general setting: Using Girsanov's theorem and coupling on the path space, the works [31, 34, 35] establish bounds on the total variation norm of such diffusions. In 4 bounds for the distance between transition probabilities of diffusions with different drifts are derived using analytic arguments, see also the related work [38]. The drawback of these approaches is that the derived bounds are typically only useful for small

1991 Mathematics Subject Classification. 60J60, 60H10.

Key words and phrases. Diffusion process, reflection coupling, sticky boundary conditions, stochastic stability, perturbations of Markov processes, total variation bounds, McKean-Vlasov.

Financial support from the German Science foundation through the Hausdorff Center for Mathematics is gratefully acknowledged. 
time horizons and are not long-time stable. The article [3] provides bounds for the distance between stationary measures of diffusions with different drifts. Coupling methods are used in [11] to provide long-time stable bounds on the distance between a Langevin diffusion and its Euler approximation. Howitt constructs in [26] a sticky coupling of two one-dimensional Brownian motions with different drifts using time-change arguments which are restricted to the one-dimensional setting.

In this article, we discuss a novel approach of constructing couplings $\left(X_{t}, Y_{t}\right)$ of solutions to (1) and (2) in a multi-dimensional setting. Consider for example the case where $\tilde{b}$ differs from $b$ by a non-zero constant $m$, i.e., $\tilde{b}(t, x)=b(t, x)+m$ for some $m \in \mathbb{R}^{d}$, and let $\left(X_{t}\right)$ and $\left(Y_{t}\right)$ be solutions of (1) and (2) respectively. In this case, whenever $X_{t}$ and $Y_{t}$ meet, the drift forces the processes to immediately move apart from each other. It is clear that, regardless of how the processes are coupled, one cannot hope for the existence of an almost surely finite stopping time $T$ such that $P\left[X_{t}=Y_{t} \forall t \geq T\right]=1$. Nevertheless, we construct a coupling such that for any given $t>0$, we have $P\left[X_{t}=Y_{t}\right]>0$ and the coupling is sticky in the sense that there is a continuous semimartingale $\left(r_{t}\right)$ which solves a one-dimensional stochastic differential equation with a sticky boundary behavior at zero such that almost surely $\left|X_{t}-Y_{t}\right| \leq r_{t}$ for all $t \geq 0$. This allows us to establish explicit, nonasymptotic and long-time stable bounds for the probability of the event $\left\{X_{t}=Y_{t}\right\}$. The coupling is constructed as a weak limit of Markovian couplings. The idea for the coupling is based on [14, 12] where coupling approaches for particle systems and nonlinear McKean-Vlasov processes are discussed, cf. Section 2.2 for a comprehensive comparison. We show that sticky couplings can be applied effectively to provide total variation bounds between the laws of both linear and non-linear diffusions with varying drifts.

Outline: The main results are presented in Section 2. In Section 3 we recall results on the existence and uniqueness of one-dimensional SDEs with sticky boundary, we establish an approximation result for the latter, and we study the long-time behavior of solutions to such equations using coupling methods. Based on these results, the proof of our main theorem and the construction of the sticky coupling are presented in Section 4 .

\section{MAin Results}

2.1. Sticky couplings. We impose the following assumptions:

Assumption 1. There is a constant $M \in[0, \infty)$ such that

$$
|b(t, x)-\tilde{b}(t, x)| \leq M \quad \text { for any } x \in \mathbb{R}^{d} \text { and } t \geq 0 .
$$

Assumption 2. There is a Lipschitz function $\kappa:[0, \infty) \rightarrow \mathbb{R}$ such that

$$
\langle x-y, b(t, x)-b(t, y)\rangle \leq \kappa(|x-y|) \cdot|x-y|^{2} \quad \text { for any } x, y \in \mathbb{R}^{d} \text { and } t \geq 0 .
$$

Outside of a bounded interval, the function $\kappa$ is constant and strictly negative.

The assumptions imply in particular that the unique strong solutions $\left(X_{t}\right)$ and $\left(Y_{t}\right)$ of (1) and (2) respectively are non-explosive. We present our main result: 


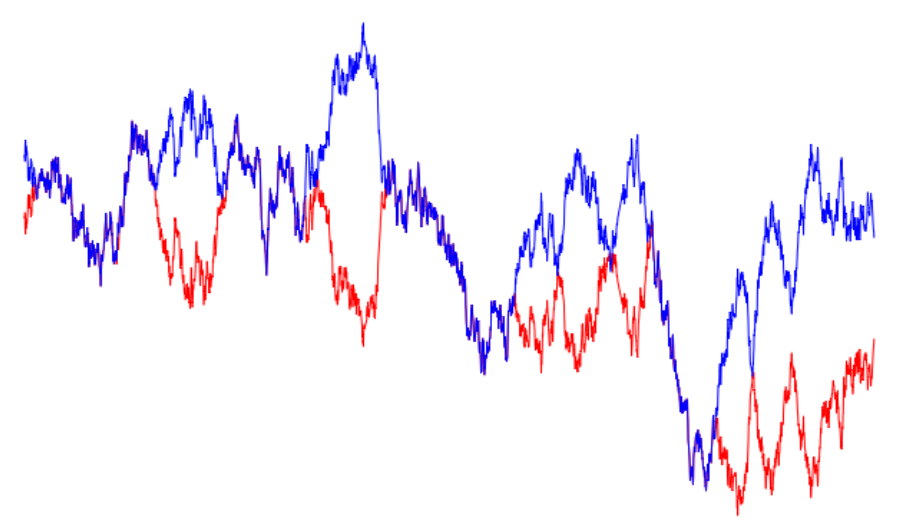

Figure 1. A sticky coupling of two diffusions on $\mathbb{R}^{1}$

Theorem 1 (Sticky coupling). Suppose that Assumptions 1 and 2 hold true. Then for any initial values $x, y \in \mathbb{R}^{d}$, there is a coupling $\left(X_{t}, Y_{t}\right)$ of solutions to (1) and (2), respectively, such that $X_{t}-Y_{t}$ is sticky at zero in the sense that the difference is controlled by a solution of a one-dimensional SDE with a sticky boundary behavior at zero. More precisely, there is a real-valued process $\left(r_{t}\right)$ solving the SDE

$$
d r_{t}=\left(M+\kappa\left(r_{t}\right) r_{t}\right) d t+2 I\left(r_{t}>0\right) d W_{t}, \quad r_{0}=|x-y|,
$$

driven by a one-dimensional Brownian motion $\left(W_{t}\right)$, such that almost surely,

$$
\left|X_{t}-Y_{t}\right| \leq r_{t} \quad \text { for any } t \geq 0 \text {. }
$$

The process $\left(r_{t}\right)$ is sticky at zero in the sense that almost surely,

$$
2 M \int_{0}^{t} I\left(r_{s}=0\right) d s=\ell_{t}^{0}(r), \quad 0 \leq t<\infty,
$$

where $\ell_{t}^{0}(r)$ is the right local time at 0 of $\left(r_{t}\right)$, i.e.,

$$
\ell_{t}^{0}(r)=\lim _{\epsilon \downarrow 0} \frac{1}{\epsilon} \int_{0}^{t} I\left(0 \leq r_{s}<\epsilon\right) d[r]_{s}=4 \lim _{\epsilon \downarrow 0} \frac{1}{\epsilon} \int_{0}^{t} I\left(0<r_{s}<\epsilon\right) d s .
$$

Equation (3) admits an invariant probability measure $\pi$. For $M=0, \pi=\delta_{0}$, and for $M>0, \pi$ is determined by

(6) $\pi(d x) \propto\left(\frac{2}{M} \delta_{0}(d x)+\exp \left(\frac{1}{2} \int_{0}^{x}(M+\kappa(y) y) d y\right) \lambda_{(0, \infty)}(d x)\right)$.

If the initial conditions coincide, i.e., if $x=y$, then for any $t \geq 0$,

(7) $P\left[X_{t}=Y_{t}\right] \geq \pi[\{0\}]=\left(1+\frac{M}{2} \int_{0}^{\infty} \exp \left(\frac{1}{2} \int_{0}^{x}(M+\kappa(y) y) d y\right) d x\right)^{-1}$.

In general, there are constants $c, \epsilon \in(0, \infty)$, depending only on $M$ and $\kappa$, such that for any $t>0$ and any initial values $x, y \in \mathbb{R}^{d}$,

$$
P\left[X_{t} \neq Y_{t}\right] \leq \frac{1}{\epsilon} \frac{c}{e^{c t}-1}|x-y|+\pi[(0, \infty)]
$$


The constants $c$ and $\epsilon$ are given by

$$
c=\left(2 \int_{0}^{R_{1}} \frac{\Phi(s)}{\varphi(s)} d s\right)^{-1} \quad \text { and } \epsilon=\min \left\{\left(2 \int_{0}^{R_{1}} \frac{1}{\varphi(s)} d s\right)^{-1}, c \Phi\left(R_{1}\right)\right\} \text {, }
$$

where $\varphi(r)=\exp \left(-\frac{1}{2} \int_{0}^{r}(M+\kappa(s) s)^{+} d s\right), \Phi(r)=\int_{0}^{r} \varphi(s) d s$,

(9) $\quad R_{0}=\inf \{R \geq 0:(M+\kappa(r) r) \leq 0$ for any $r \geq R\}$, and

(10) $R_{1}=\inf \left\{R \geq R_{0}: R\left(R-R_{0}\right)(M / r+\kappa(r)) \leq-4\right.$ for any $\left.r \geq R\right\}$.

In Section 3 we also provide explicit bounds on the expected values $E\left[\left|X_{t}-Y_{t}\right|\right]$, cf. Theorem 5 further below.

The coupling $\left(X_{t}, Y_{t}\right)$ in Theorem 1 is constructed as a weak limit of Markovian couplings. The construction of the coupling and the proof of the theorem are given in Section 4 .

Remark 1 (Reflection coupling). The classical reflection coupling of Lindvall and Rogers [36] occurs as a special case of the coupling in Theorem 1 when the drift coefficients coincide, i.e., $b=\tilde{b}$. In this case we can choose $M=0$ so that 0 is an absorbing boundary for the diffusion process $\left(r_{t}\right)$. The equation (11) reduces to

$$
P\left[X_{t} \neq Y_{t}\right] \leq \frac{1}{\epsilon} \frac{c}{e^{c t}-1}|x-y|,
$$

which is a well-known bound for reflection coupling [36, 7].

In the two special cases $M=0$ and $x=y$, the bound in (11) takes a very simple and intuitive form. In general, however, the rate $c$ depends on $M$. This dependence can be avoided by considering a modified coupling.

Theorem 2. There is a coupling $\left(\tilde{X}_{t}, \tilde{Y}_{t}\right)$ of solutions to (1) and (2) such that

$$
P\left[\tilde{X}_{t} \neq \tilde{Y}_{t}\right] \leq \frac{1}{\tilde{\epsilon}} \frac{\tilde{c}}{e^{\tilde{c} t}-1}|x-y|+\pi[(0, \infty)] \quad \text { for any } t \geq 0,
$$

where $\tilde{c}, \tilde{\epsilon}$ are defined analogously to $c$ and $\epsilon$ but with $M=0$.

Proof of Theorem 2. Consider a process $\left(Z_{t}\right)$ satisfying

$$
d Z_{t}=b\left(t, Z_{t}\right) d t+d B_{t}, \quad Z_{0}=y .
$$

Let $\left(\tilde{X}_{t}, \tilde{Z}_{t}\right)$ be a standard reflection coupling of $\left(X_{t}\right)$ and $\left(Z_{t}\right)$, i.e., a sticky coupling in the case where the drifts coincide. Then we can glue this coupling with a sticky coupling of $\left(Z_{t}\right)$ and $\left(Y_{t}\right)$, i.e., there are processes $\left(\tilde{X}_{t}, \tilde{Z}_{t}, \tilde{Y}_{t}\right)$ defined on a joint probability space such that $\left(\tilde{X}_{t}, \tilde{Z}_{t}\right)$ is a sticky coupling of $\left(X_{t}, Z_{t}\right)$, and $\left(\tilde{Z}_{t}, \tilde{Y}_{t}\right)$ is a sticky coupling of $\left(Z_{t}, Y_{t}\right)$, see e.g. the "glueing lemma" in [50]. For $t \geq 0$, we obtain by Theorem 1 ;

$$
P\left[\tilde{X}_{t} \neq \tilde{Y}_{t}\right] \leq P\left[\tilde{X}_{t} \neq \tilde{Z}_{t}\right]+P\left[\tilde{Z}_{t} \neq \tilde{Y}_{t}\right] \leq \frac{1}{\tilde{\epsilon}} \frac{\tilde{c}}{e^{\tilde{c} t}-1}|x-y|+\pi[(0, \infty)] .
$$


To make the bounds in the theorems more explicit, we now assume that we are given constants $\mathcal{R}, L \in[0, \infty)$ and $K \in(0, \infty)$ such that for any $t \geq 0$,

$$
\langle x-y, b(t, x)-b(t, y)\rangle \leq \begin{cases}L|x-y|^{2} & \text { for any } x, y \in \mathbb{R}^{d} \\ -K|x-y|^{2} & \text { for } x, y \in \mathbb{R}^{d} \text { s.t. }|x-y| \geq \mathcal{R} .\end{cases}
$$

Hence Assumption 2 is satisfied with $\kappa(r)=L I(r<\mathcal{R})-K I(r \geq \mathcal{R})$. In this case, the exponential decay rate $\tilde{c}$ in Theorem 2 is bounded from below by

$$
\tilde{c}^{-1} \leq \begin{cases}4 \max \left(\mathcal{R}^{2}, K^{-1}\right) & \text { if } L=0, \\ 3 e \max \left(\mathcal{R}^{2}, 4 K^{-1}\right) & \text { if } L \mathcal{R}^{2} \leq 4 \\ 8 \sqrt{\pi} L^{-1 / 2}\left(L^{-1}+K^{-1}\right) \mathcal{R}^{-1} \exp \left(L \mathcal{R}^{2} / 4\right)+16 K^{-2} \mathcal{R}^{-2} & \text { if } L \mathcal{R}^{2}>4\end{cases}
$$

see Lemma 1 in [14] (Note that the definitions of the function $\kappa$ and the constant $c$ in [14] differ from the definitions above by a factor $-2,2$, respectively). The following lemma provides explicit upper bounds on the long-time asymptotics of the probabilities in (11) and (12). The proof is included in Section 4 .

Lemma 1. Suppose that Condition (13) is satisfied. Then $\pi[(0, \infty)]=\alpha /(1+\alpha)$ where $\alpha$ is a non-negative constant such that for $M \leq K \mathcal{R}$,

$$
\alpha \leq\left(\pi^{1 / 2} e^{1 / 2} K^{-1 / 2}+2 \mathcal{R} \max \left(4, L \mathcal{R}^{2}+2 M \mathcal{R}\right)^{-1}\right) M \exp \left(M \mathcal{R} / 2+L \mathcal{R}^{2} / 4\right),
$$

and for $M \geq K \mathcal{R}$,

$$
\alpha \leq\left(\sqrt{\frac{\pi}{K}}+\frac{2 \mathcal{R}}{\max \left(4,2 M \mathcal{R}+L \mathcal{R}^{2}\right)}\right) M \exp \left(\frac{M^{2}}{4 K}+\frac{L+K}{4} \mathcal{R}^{2}\right) .
$$

The theorems imply bounds on the total variation distance between the laws of $X_{t}$ and $Y_{t}$ for any time $t \geq 0$. We now verify that in two simple examples, the bound in $(12)$ is of the correct order:

Example 1 (Ornstein-Uhlenbeck processes). Fix $m \in \mathbb{R}^{d} \backslash\{0\}$. We consider Ornstein-Uhlenbeck processes on $\mathbb{R}^{d}$, given by

$$
\begin{aligned}
d X_{t} & =-X_{t} / 2 d t \quad+d B_{t}, & X_{0} & =x, \\
d Y_{t} & =-\left(Y_{t}-m\right) / 2 d t+d \tilde{B}_{t}, & Y_{0} & =y,
\end{aligned}
$$

where $\left(B_{t}\right)$ and $\left(\tilde{B}_{t}\right)$ are $d$-dimensional Brownian motions. Let $d(t)$ denote the total variation distance between the laws of $X_{t}$ and $Y_{t}$ at time $t$. It is well-known that $X_{t}$ and $Y_{t}$ are normally distributed with

$$
\begin{aligned}
\operatorname{Law}\left(X_{t}\right) & =\mathcal{N}\left(e^{-t / 2} x,\left(1-e^{-t}\right) \mathrm{I}_{d}\right) \\
\operatorname{Law}\left(Y_{t}\right) & =\mathcal{N}\left(e^{-t / 2} y+\left(1-e^{-t / 2}\right) m,\left(1-e^{-t}\right) \mathrm{I}_{d}\right) .
\end{aligned}
$$

The total variation distance between $d$-dimensional normal distributions $\mathcal{N}\left(a, b I_{d}\right)$ and $\mathcal{N}\left(\tilde{a}, b I_{d}\right)$ with $a, \tilde{a} \in \mathbb{R}^{d}$ and $b \in(0, \infty)$ is given by $\Phi_{1}(|a-\tilde{a}| /(2 \sqrt{b}))$ where

$$
\Phi_{1}(r):=\sqrt{2 / \pi} \int_{0}^{r} \exp \left(-x^{2} / 2\right) d x
$$


cf. e.g. [10, Exercise 15.12]. Hence for any $t>0$,

$$
d(t)=\left\|\operatorname{Law}\left(X_{t}\right)-\operatorname{Law}\left(Y_{t}\right)\right\|_{T V}=\Phi_{1}\left(\frac{\left|m+e^{-t / 2}(y-m-x)\right|}{2 \sqrt{1-e^{-t}}}\right)
$$

We now compare the upper bound $(12)$ for the total variation distance that has been derived by sticky couplings to the exact expression (16). Observe that Assumptions 11 and 2 are satisfied with $M=|m| / 2$ and the constant function $\kappa(r)=-1 / 2$ respectively. By a straightforward computation we obtain

$$
\pi[(0, \infty)]=1-\left(1+\sqrt{\pi / 8}|m| e^{m^{2} / 8}\left(1+\Phi_{1}(|m| / 2)\right)\right)^{-1} .
$$

Asymptotically as $t \rightarrow \infty$, the upper bound for $P\left[\tilde{X}_{t} \neq \tilde{Y}_{t}\right]$ in (12) approaches (17), whereas the total variation distance $d(t)$ converges to $\Phi_{1}(|m| / 2)$. Comparing both expressions for small and large values of $|m|$, we see that as $|m| \rightarrow 0$,

$$
\pi[(0, \infty)] \sim \sqrt{\pi / 8}|m|, \quad \text { whereas } \Phi_{1}(|m| / 2) \sim|m| / \sqrt{2 \pi},
$$

and as $|m| \rightarrow \infty$,

$$
1-\pi[(0, \infty)] \sim \frac{2}{\sqrt{2 \pi}|m|} e^{-|m|^{2} / 8}, \quad \text { whereas } \quad 1-\Phi_{1}(|m| / 2) \sim \frac{4}{\sqrt{2 \pi}|m|} e^{-|m|^{2} / 2} .
$$

Hence as $m \downarrow 0$, the bounds for the long time limit of the total variation distance provided by sticky couplings are of the correct order up to a multiplicative constant, whereas for $m \rightarrow \infty$, we loose a factor 4 in the exponential.

Furthermore, we can compare the decay rate $\tilde{c}$ in $(12)$ with the rate of convergence of $d(t)$ to its limit $\Phi_{1}(|m| / 2)$. Asymptotically as $t \uparrow \infty, 16$ implies

$$
\begin{aligned}
\left|d(t)-\Phi_{1}(|m| / 2)\right| & \sim \Phi_{1}^{\prime}(|m| / 2) e^{-t / 2}|y-m-x| / 2 \\
& =(2 \pi)^{-1 / 2} e^{-m^{2} / 8} e^{-t / 2}|y-m-x| .
\end{aligned}
$$

On the other hand, in this case $\tilde{c}=1 / 8$ and $\tilde{\epsilon}=1 /(2 \sqrt{8})$, so by 12 ,

$$
P\left[\tilde{X}_{t} \neq \tilde{Y}_{t}\right]-\pi[(0, \infty)] \leq 2^{-1 / 2}\left(e^{t / 8}-1\right)^{-1}|x-y|
$$

We see that the exponential rate of decay in our bound differs from the optimal rate only by a factor 4 .

Example 2 (Confined Brownian motion). Fix $R, k, m \in(0, \infty)$, and let

$$
b(x)=0 \text { for }|x| \leq R, \quad \text { and } \quad b(x)=-k(x-R \operatorname{sgn}(x)) / 2 \text { otherwise. }
$$

Moreover, let $\tilde{b}(x)=b(x)+m / 2$. In this case, Condition (13) is satisfied with $L=0, K=k / 6$ and $\mathcal{R}=3 R$, and Assumption 1 holds with $M=m / 2$. Assuming $m \leq k R$ and $m R \leq 4 / 3$, Theorem 2 and the first bound in Lemma 1 show that there is a coupling $\left(\tilde{X}_{t}, \tilde{Y}_{t}\right)$ of the corresponding solutions to (1) and (2) with arbitrary initial values $x$ and $y$ such that

$$
\limsup _{t \rightarrow \infty} P\left[\tilde{X}_{t} \neq \tilde{Y}_{t}\right] \leq\left(\frac{3 e}{4} R+\left(3 \pi e^{3} / 2\right)^{1 / 2} k^{-1 / 2}\right) m .
$$

On the other hand, the unique invariant probability measures for (1) and (2) are given explicitly by $\nu(d x)=Z_{f}^{-1} f(x) d x, \mu(d x)=Z_{g}^{-1} g(x) d x$, respectively, where 
$f(x)=\exp \left(-k \max (|x|-R, 0)^{2} / 2\right), g(x)=\exp (m x) f(x), Z_{f}=\int_{-\infty}^{\infty} f(x) d x$ and $Z_{g}=\int_{-\infty}^{\infty} g(x) d x$. Noting that $Z_{g} \geq Z_{f}$, an explicit computation yields the lower bounds

$$
\|\mu-\nu\|_{T V} \geq(\exp (-m R)-1+m R) /(m R),
$$

and, for $R k^{1 / 2} \leq 1$,

$$
\|\mu-\nu\|_{T V} \geq\left(1-\exp \left(-m R+m^{2} /(2 k)\right)+2^{1 / 2}(\pi k)^{-1 / 2} m \exp (-m R)\right) / 4,
$$

see the appendix. In particular,

$$
\liminf _{m \downarrow 0}\|\mu-\nu\|_{T V} / m \geq \frac{1}{4}\left(R+(2 / \pi)^{1 / 2} k^{-1 / 2}\right) .
$$

Hence for small $m$, the bound in $(20)$ is sharp up to a constant factor.

Remark 2 (Comparison with Girsanov couplings). An alternative approach to construct couplings of solutions to (1) and (2) is by Girsanov's Theorem. If the initial conditions $X_{0}$ and $Y_{0}$ coincide and $T \in[0, \infty)$ is a fixed constant, then Girsanov's Theorem can be applied to construct a coupling $\left(X_{s}, Y_{s}\right)$ such that with positive probability, $X_{s}=Y_{s}$ for all $s \in[0, T]$. Moreover, explicit bounds on this probability can be derived via Hellinger integrals [31, 34, 35]. Notice, however, that the corresponding bounds typically degenerate rapidly as $T \rightarrow \infty$. Hence Girsanov's Theorem provides a very strong coupling over short time intervals, whereas the sticky couplings introduced above are stable for long times in the sense that $\liminf _{t \rightarrow \infty} P\left[X_{t}=Y_{t}\right] \geq \pi[\{0\}]>0$.

2.2. McKean-Vlasov processes. We consider nonlinear diffusions on $\mathbb{R}^{d}$ of type

$$
\begin{aligned}
d X_{t} & =\eta\left(X_{t}\right) d t+\tau \int \vartheta\left(X_{t}, y\right) \mu_{t}^{x}(d y) d t+d B_{t}, \quad X_{0}=x, \\
\mu_{t}^{x} & =\operatorname{Law}\left(X_{t}\right),
\end{aligned}
$$

where $\left(B_{t}\right)$ is a $d$-dimensional Brownian motion and $\tau \in \mathbb{R}$. The SDE is nonlinear in the sense of McKean, i.e., the future development after time $t$ depends on the current state $X_{t}$ and on the law of $X_{t}$, cf. e.g. [49, 40. Let $\eta: \mathbb{R}^{d} \rightarrow \mathbb{R}^{d}$ and $\vartheta: \mathbb{R}^{d} \times \mathbb{R}^{d} \rightarrow \mathbb{R}^{d}$ be Lipschitz continuous functions. Then the equation above admits a unique strong solution, cf. [40, Theorem 2.2]. Let us fix initial values $x_{0}, y_{0} \in \mathbb{R}^{d}, x_{0} \neq y_{0}$, and consider solutions $\left(X_{t}\right)$ and $\left(Y_{t}\right)$ of 21 with $X_{0}=x_{0}$ and $Y_{0}=y_{0}$ respectively. We define drift coefficients

$$
\begin{aligned}
& b^{x_{0}}(t, x)=\eta(x)+\tau \int \vartheta(x, y) \mu_{t}^{x_{0}}(d y), \\
& b^{y_{0}}(t, x)=\eta(x)+\tau \int \vartheta(x, y) \mu_{t}^{y_{0}}(d y),
\end{aligned}
$$

which are uniformly Lipschitz in $x$ and continuous in $t$. Notice that due to pathwise uniqueness, $\left(X_{t}\right)$ and $\left(Y_{t}\right)$ are the unique strong solutions to the equations

$$
\begin{aligned}
d X_{t} & =b^{x_{0}}\left(t, X_{t}\right) d t+d B_{t}, & & X_{0}=x_{0}, \\
d Y_{t} & =b^{y_{0}}\left(t, Y_{t}\right) d t+d B_{t}, & & Y_{0}=y_{0},
\end{aligned}
$$

and hence we can interpret the processes as two diffusions with different drifts. 
Assumption 3. There is a Lipschitz function $\kappa:[0, \infty) \rightarrow \mathbb{R}$ such that

$$
\langle x-y, \eta(x)-\eta(y)\rangle \leq \kappa(|x-y|) \cdot|x-y|^{2} \quad \text { for any } x, y \in \mathbb{R}^{d} \text { and } t \geq 0 .
$$

Outside of a bounded interval, the function $\kappa$ is constant and strictly negative.

Assuming that Assumption 3 holds, we have shown in 12 that there are constants $A, \lambda, \tau_{0} \in(0, \infty)$ such that for $|\tau| \leq \tau_{0}$,

$$
\mathcal{W}^{1}\left(\mu_{t}^{x}, \mu_{t}^{y}\right) \leq A e^{-\lambda t}|x-y| \text { for any } t \geq 0 \text { and } x, y \in \mathbb{R}^{d},
$$

where $\mathcal{W}^{1}$ denotes the standard $L^{1}$ Wasserstein distance. The proof is based on an application of reflection coupling if $\left|X_{t}-Y_{t}\right| \geq \delta$ and synchronous coupling if $\left|X_{t}-Y_{t}\right| \leq \delta / 2$, where $\delta$ is a small positive constant. In the intermediate region, a combination of both couplings is applied. The bound in $(26)$ is obtained when considering the limit of the resulting bounds as $\delta \downarrow 0$. The couplings considered in [12] now turn out to be approximations of a sticky coupling. By applying directly the sticky coupling and using Corollary 1 further below, we can extend the result in [12 and derive a corresponding exponential decay in total variation norm:

Theorem 3. Let $\eta$ and $\vartheta$ be Lipschitz and let Assumption 3 be true. There is $\tau_{0} \in(0, \infty)$ such that for any $|\tau| \leq \tau_{0}$ and any $x, y \in \mathbb{R}^{d}$ there are constants $B, c \in(0, \infty)$ such that,

$$
\left\|\mu_{t}^{x}-\mu_{t}^{y}\right\|_{T V} \leq B e^{-c t} \text { for any } t \geq 0 .
$$

The proof is given in Section 4 .

2.3. Outlook. The concept of sticky couplings sheds new light onto several results that have been previously derived using combinations of reflection and synchronous couplings. A first example of this type has been given in Theorem 3 . Without carrying out details, we mention three further results that probably can be reinterpreted in terms of sticky couplings:

a) Componentwise reflection couplings for interacting diffusions. In [14], Wasserstein bounds for interacting diffusions with small interaction term (for example of mean-field-type) have been derived by coupling each component independently with a reflection coupling if the distance is greater than a given constant $\delta>0$, and with a synchronous coupling otherwise. Instead, one could now directly consider a componentwise sticky coupling. As time evolves, more and more components in this coupling would get stuck at nearby positions until, after some finite coupling time, all components coincide. We expect that such a coupling could be used to derive total variation bounds similar to those in Theorem 3 for interacting particle systems.

b) Couplings for infinite-dimensional diffusions. In [54], Wasserstein contraction rates have been derived for a class of diffusions on a Hilbert space with possibly degenerate noise. Here a reflection coupling has been applied to the projection of the process on a finite dimensional subspace, whereas the remaining (orthogonal) components have been coupled synchronously. Again, because of the interaction between the components, the reflection coupling is switched off when the finite dimensional projections of the two copies are close to each other. Similarly as above, 
it should be possible to replace the coupling for the finite dimensional projection by a sticky coupling. The resulting infinite dimensional coupling process would then spend a certain amount of time at states where the finite dimensional projections of the two copies coincide. Under the assumptions made in [54, the orthogonal infinite dimensional components would approach each other for large $t$, and, consequently, the finite dimensional projections would coincide for an increasing proportion of time.

c) Couplings for Langevin processes. In a forthcoming paper, we consider couplings for (kinetic) Langevin diffusions $\left(X_{t}, V_{t}\right)_{t \geq 0}$ with state space $\mathbb{R}^{2 d}$ that are given by stochastic differential equations of type

$$
\begin{aligned}
d X_{t} & =V_{t} d t \\
d V_{t} & =-\gamma V_{t} d t-u \nabla U\left(X_{t}\right) d t+\sqrt{2 \gamma u} d B_{t} .
\end{aligned}
$$

Here $\left(B_{t}\right)_{t \geq 0}$ is a $d$ dimensional Brownian motion, $u$ and $\gamma$ are positive constants, and $U$ is a $C^{1}$ function on $\mathbb{R}^{d}$. We apply a reflection coupling that is replaced by a synchronous coupling when the values of $X_{t}+\gamma^{-1} V_{t}$ are close to each other for both components. Again, at least informally, this coupling could be replaced by a coupling $\left(\left(X_{t}, V_{t}\right),\left(X_{t}^{\prime}, V_{t}^{\prime}\right)\right)$ that is sticky when $X_{t}+\gamma^{-1} V_{t}=X_{t}^{\prime}+\gamma^{-1} V_{t}^{\prime}$. Under the assumptions that we impose on $U$, the coupling would be contractive on the corresponding $3 d$ dimensional linear subspace of $\mathbb{R}^{4 d}$, and as time evolves, it would spend a positive amount of time on this subspace.

We hope that the potential applications listed above show how sticky couplings provide a valuable concept for building intuition about ways to couple diffusion processes in an efficient way. Carrying out carefully the ideas described above would go far beyond the scope of this paper.

\section{Diffusions on $\mathbb{R}_{+}$With A STICKY REFlECting Boundary.}

In this section we prove some basic results on diffusions on $\mathbb{R}_{+}$with a sticky boundary at 0 . In particular, we prove the existence of a synchronous coupling of two sticky diffusions and a corresponding comparison theorem, which is then applied to study the long time behavior of the processes. At first, we need to adapt some known facts on existence and uniqueness of weak solutions to our setup. We consider the stochastic differential equation

$$
d r_{t}=\alpha\left(t, r_{t}\right) d t+2 I\left(r_{t}>0\right) d W_{t}, \quad \operatorname{Law}\left(r_{0}\right)=\mu,
$$

on the positive real line $\mathbb{R}_{+}=[0, \infty)$, where $\left(W_{t}\right)$ is a one-dimensional Brownian motion and $\mu$ is a probability measure on $\mathbb{R}_{+}$. Below, we will impose conditions on the drift coefficient $\alpha: \mathbb{R}_{+} \times \mathbb{R}_{+} \rightarrow \mathbb{R}$ which imply existence and uniqueness of weak solutions. In particular, we will assume that $\alpha(t, 0)>0$ for any $t \geq 0$. Let us briefly discuss the consequences of this assumption: Suppose that $\left(r_{t}\right)$ is a solution of (29). An application of the Itô-Tanaka formula to $f\left(r_{t}\right)$ with the function $f(x)=\max (0, x)$ and a comparison with 29 shows that almost surely,

$$
\int_{0}^{t} \alpha(s, 0) I\left(r_{s}=0\right) d s=\frac{1}{2} \ell_{t}^{0}(r), \quad 0 \leq t<\infty
$$


where $\ell_{t}^{0}(r)=\lim _{\epsilon \downarrow 0} \epsilon^{-1} \int_{0}^{t} I\left(0 \leq r_{s} \leq \epsilon\right) d[r]_{s}$ is the right local time of $\left(r_{t}\right)$. Equation (30) shows that there is reflection at zero. Moreover, for almost all trajectories, the Lebesgue measure of the set $\left\{0 \leq s \leq t: r_{s}=0\right\}$ increases whenever $\ell_{t}^{0}(r)$ increases. In this sense $\left(r_{t}\right)$ is sticky at zero.

Stochastic differential equations with boundary conditions have a long history. The discovery of a sticky boundary behavior for one-dimensional diffusions seems to go back to Feller [17, 18]. A historical overview is given in [41]. We give references to the most relevant works for our application and some recent developments. Existence and uniqueness results for multi-dimensional diffusion processes with various boundary behaviors have been established by Ikeda and Watanabe in [27, 52, 53. These are based on results by Skorokhod and McKean [45, 46, 39]. Martingale problems with boundary conditions have been investigated by Stroock and Varadhan [47, see also the related work [19]. Non-existence of a strong solution to the SDE for sticky Brownian motion has been established in [8]. In [51], Warren identifies the law of a sticky Brownian motion conditioned on the driving Wiener process, see also the related work [22]. A recent publication on existence and uniqueness, which is also a good introduction into the topic, is the work by Engelbert and Peskir [15] and the related work [2]. First steps towards sticky couplings in a one-dimensional setting have been made by Howitt in [26] based on time-changes. The recent articles [20, 21] use Dirichlet forms to investigate sticky diffusions and provide some ergodicity results. Rácz and Shkolnikov [44] construct a multi-dimensional sticky Brownian motion as a limit of exclusion processes, see also [1] and [23].

3.1. Existence, uniqueness and comparison of solutions. We use the concept of weak solutions. Let $\left(\Omega, \mathcal{A},\left(\mathcal{F}_{t}\right), P\right)$ be a filtered probability space satisfying the usual conditions. An $\left(\mathcal{F}_{t}\right)$ adapted process $\left(r_{t}, W_{t}\right)$ on $(\Omega, \mathcal{A}, P)$ is called a weak solution of (29) if $P \circ r_{0}^{-1}=\mu,\left(W_{t}\right)$ is a one-dimensional $\left(\mathcal{F}_{t}\right)$-Brownian motion w.r.t. $P$, and $\left(r_{t}\right)$ is continuous, non-negative, and $P$-almost surely,

$$
r_{t}-r_{0}=\int_{0}^{t} \alpha\left(s, r_{s}\right) d s+\int_{0}^{t} 2 I\left(r_{s}>0\right) d W_{s}, \quad 0 \leq t<\infty .
$$

We will make the following assumptions on the drift coefficient:

Assumption 4. For any $R>0$, $\inf _{t \in[0, R]} \alpha(t, 0)>0$.

Assumption 5. For any $R>0$ there is $L_{R} \in(0, \infty)$ such that

$$
|\alpha(t, x)-\alpha(s, y)| \leq L_{R}(|t-s|+|x-y|) \quad \text { for any } x, y, s, t \in[0, R] .
$$

Assumption 6. There is $C \in(0, \infty)$ such that for any $x \in \mathbb{R}_{+}$,

$$
\sup _{t \in[0, \infty)} \alpha(t, x) \leq C(1+|x|)
$$

The assumptions above imply existence and uniqueness in law of weak solutions to (29). This has been proven by Watanabe in [52, 53] assuming that the maps $(t, x) \mapsto \alpha(t, x)$ and $t \mapsto 1 / \alpha(t, 0)$ are bounded and Lipschitz. Using localization techniques for martingale problems, following the work of Stroock and Varadhan [48], Watanabe's results can be transferred to our slightly more general setup: 
3.1.1. Uniqueness in law. Let $\mathbb{W}=C\left(\mathbb{R}_{+}, \mathbb{R}\right)$ be the space of continuous functions endowed with the topology of uniform convergence on compacts, and let $\mathcal{B}(\mathbb{W})$ denote the Borel $\sigma$-Algebra. Let $\mathcal{F}_{t}=\sigma\left(\boldsymbol{r}_{s}: 0 \leq s \leq t\right)$ be the natural filtration generated by the canonical process $\boldsymbol{r}_{t}(\omega)=\omega(t)$. Given a solution $\left(r_{t}\right)$ of $(29)$, defined on a probability space $(\Omega, \mathcal{A}, P)$, we write $\mathbb{P}=P \circ r^{-1}$ for the law of $r$ on $(\mathbb{W}, \mathcal{B}(\mathbb{W}))$. We say that solutions to $(29)$ are unique in law, if any two solutions $\left(r_{t}^{1}\right)$ and $\left(r_{t}^{2}\right)$ with coinciding initial law have the same law on the space $(\mathbb{W}, \mathcal{B}(\mathbb{W}))$.

In order to apply existing localization techniques for martingale problems, we interpret equation $(29)$ as an equation on $\mathbb{R}$, instead of $\mathbb{R}_{+}$, setting $\alpha(t, x)=\alpha(t, 0)$ for $x<0$. This does not cause any problems since, under the assumptions imposed above, any solution $\left(r_{t}\right)$ with initial law supported on $\mathbb{R}_{+}$satisfies almost surely $r_{t} \geq 0$ for all $t \geq 0$, see e.g. the argument in [15, Proof of Theorem 5].

We follow [48, 32] and define a family of second order differential operators

$$
\left(\mathcal{L}_{t} f\right)(x)=\alpha(t, x) f^{\prime}(x)+(1 / 2) I(x>0) f^{\prime \prime}(x) .
$$

A probability measure $\mathbb{P}$ on $(\mathbb{W}, \mathcal{B}(\mathbb{W}))$ is called a solution to the martingale problem w.r.t. $\left(\mathcal{L}_{t}\right)$ iff for any $f \in C_{0}^{2}(\mathbb{R})$,

$$
M_{t}^{f}=f\left(\boldsymbol{r}_{t}\right)-f\left(\boldsymbol{r}_{0}\right)-\int_{0}^{t}\left(\mathcal{L}_{u} f\right)\left(\boldsymbol{r}_{u}\right) d u
$$

is a continuous $\left(\mathcal{F}_{t}\right)$-martingale under $\mathbb{P}$. The solution to the martingale problem is called unique, if any two solutions $\mathbb{P}^{1}$ and $\mathbb{P}^{2}$ coincide whenever $\mathbb{P}^{1} \circ \boldsymbol{r}_{0}^{-1}=\mathbb{P}^{2} \circ \boldsymbol{r}_{0}^{-1}$. The next two results are well-known:

Lemma 2. [48, 32] The following statements are equivalent:

(i) There is a weak solution of $(29)$ with initial distribution $\mu$.

(ii) There is a solution $\mathbb{P}$ to the martingale problem w.r.t. $\left(\mathcal{L}_{t}\right)$ s.t. $\mathbb{P} \circ \boldsymbol{r}_{0}^{-1}=\mu$. Moreover, the uniqueness of solutions to the martingale problem w.r.t. $\left(\mathcal{L}_{t}\right)$ and the uniqueness in law of weak solutions to (29) are equivalent.

Lemma 3. [52, 53] Assume that the maps $(t, x) \mapsto \alpha(t, x)$ and $t \mapsto 1 / \alpha(t, 0)$ are bounded and Lipschitz. Then for any initial law $\mu$ on $\mathbb{R}_{+}$, there is a weak solution to 29) which is unique in law.

A detailed proof of Lemma 2 can be found in [32, Chapter 5, Section 4.B]. A proof of Lemma 3 is given in [29, Chapter IV, Section 7].

Lemma 4. If Assumptions 4 and 5 are satisfied then the solution to the martingale problem w.r.t. $\left(\mathcal{L}_{t}\right)$ is unique for a given initial law, and thus uniqueness in law holds for solutions to 29 .

Proof. We set $\alpha_{n}(s, x)=\alpha(s \wedge n, x \wedge n)$ for $n \in \mathbb{N}$. By the assumptions, the maps $(t, x) \mapsto \alpha_{n}(t, x)$ and $t \mapsto 1 / \alpha_{n}(t, 0)$ are bounded and Lipschitz continuous. Hence uniqueness holds for the corresponding martingale problem for any initial law $\mu$ on $\mathbb{R}_{+}$according to Lemma 3 and 2 . The uniqueness for the martingale problem w.r.t. $\left(\mathcal{L}_{t}\right)$ for such initial laws can now be shown by a localization argument, cf. [48, Theorem 10.1.2]. 
3.1.2. Approximation, existence and coupling of solutions. We now consider two equations of the form (29) with drift coefficients $\beta$ and $\gamma$ that both satisfy Assumptions 4, 5 and 6. We construct a synchronous coupling of solutions to these equations as a weak limit of solutions to approximating equations with locally Lipschitz continuous coefficients. We introduce the family of stochastic differential equations, indexed by $n \in \mathbb{N}$, given by

$$
\begin{aligned}
& d \tilde{r}_{t}^{n}=\beta\left(t, \tilde{r}_{t}^{n}\right) d t+2 \vartheta^{n}\left(\tilde{r}_{t}^{n}\right) d \tilde{W}_{t}, \quad \operatorname{Law}\left(\tilde{r}_{0}^{n}, \tilde{s}_{0}^{n}\right)=\tilde{\mu}^{n} \otimes \tilde{\nu}^{n}, \\
& d \tilde{s}_{t}^{n}=\gamma\left(t, \tilde{s}_{t}^{n}\right) d t+2 \vartheta^{n}\left(\tilde{s}_{t}^{n}\right) d \tilde{W}_{t},
\end{aligned}
$$

Here $\left(\tilde{W}_{t}\right)$ is a Brownian motion, and we assume that:

Assumption 7. $\left(\tilde{\mu}^{n}\right)$ and $\left(\tilde{\nu}^{n}\right)$ are sequences of probability measures on $\mathbb{R}_{+}$converging weakly towards probability measures $\tilde{\mu}$ and $\tilde{\nu}$, respectively.

Assumption 8. For each $n \in \mathbb{N}$, the function $\vartheta^{n}: \mathbb{R}_{+} \rightarrow[0,1]$ is Lipschitz continuous with $\vartheta^{n}(0)=0, \vartheta^{n}(x)>0$ for $x>0$, and $\vartheta^{n}(x)=1$ for $x \geq 1 / n$.

Remark 3. In [15], a sticky Brownian motion $\left(r_{t}\right)$ satisfying

$$
d r_{t}=I\left(r_{t} \neq 0\right) d \tilde{W}_{t}, \quad I\left(r_{t}=0\right) \mu d t=d \ell_{t}^{0}(r), \quad \mu \in(0, \infty)
$$

is approximated by solutions of equations

$$
d r_{t}^{n}=\left(\sqrt{2 \mu / n} I\left(\left|r_{t}^{n}\right| \leq 1 / n\right)+I\left(\left|r_{t}^{n}\right|>1 / n\right)\right) d \tilde{W}_{t},
$$

The approximation is tailored in such a way that it is compliant with the timechanges frequently used to show existence and uniqueness of weak solutions to sticky SDEs, see e.g. [15, 53. Our approximation result follows a similar spirit but it does not rely on time changes.

Lemma 5. Suppose that $\beta$ and $\gamma$ satisfy Assumptions 4 , 5 and 6 . Moreover, let Assumptions 7 and 8 be true. Then for each $n \in \mathbb{N}$, there is a strong solution $\left(\tilde{r}_{t}^{n}, \tilde{s}_{t}^{n}\right)$ of Equation (31) with values in $\mathbb{R}_{+}^{2}$. Moreover, uniqueness in law holds.

Proof. Fix $n \in \mathbb{N}$. For $x<0$ we set $\vartheta^{n}(x)=0, \beta(t, x)=\beta(t, 0)$, and $\gamma(t, x)=$ $\gamma(t, 0)$. Equation 31 is then a standard SDE on $\mathbb{R}^{2}$ with locally Lipschitz coefficients. Hence there is a strong and pathwise unique solution. Moreover, Assumption 6 implies that the solution is non-explosive. Similarly to [15, Proof of Theorem 5], we can apply the Itô-Tanaka formula to the negative part of $\tilde{r}_{t}^{n}$ in order to show that the process is non-negative. Indeed,

$$
\left(\tilde{r}_{t}^{n}\right)^{-}-\left(\tilde{r}_{0}^{n}\right)^{-}=-\int_{0}^{t} I\left(\tilde{r}_{s}^{n} \leq 0\right) d \tilde{r}_{s}^{n}+\frac{1}{2} \ell_{t}^{0}\left(\tilde{r}^{n}\right),
$$

where $\ell_{t}^{0}\left(\tilde{r}^{n}\right)$ is the right local time of $\left(\tilde{r}_{t}^{n}\right)$, i.e.,

$$
\ell_{t}^{0}\left(\tilde{r}^{n}\right)=\lim _{\epsilon \downarrow 0} \epsilon^{-1} \int_{0}^{t} I\left(0 \leq \tilde{r}_{s}^{n} \leq \epsilon\right) d\left[\tilde{r}^{n}\right]_{s}=4 \lim _{\epsilon \downarrow 0} \epsilon^{-1} \int_{0}^{t} I\left(0 \leq \tilde{r}_{s}^{n} \leq \epsilon\right) \vartheta^{n}\left(\tilde{r}_{s}^{n}\right)^{2} d s .
$$

Since $\vartheta^{n}$ is Lipschitz with $\vartheta^{n}(0)=0$, the local time vanishes. Therefore, and since $\beta(s, 0)>0$ for any $s \geq 0$, we have $0 \leq\left(\tilde{r}_{t}^{n}\right)^{-} \leq\left(\tilde{r}_{0}^{n}\right)^{-}=0$. A similar argument can be used for $\left(\tilde{s}_{t}^{n}\right)$. 
For each $n \in \mathbb{N}$, there are a probability space $\left(\Omega^{n}, \mathcal{A}^{n}, P^{n}\right)$ and random variables $\tilde{r}^{n}, \tilde{s}^{n}: \Omega^{n} \rightarrow \mathbb{W}$ such that $\left(\tilde{r}_{t}^{n}, \tilde{s}_{t}^{n}\right)$ is a solution of $(31)$. Let $\mathbb{P}^{n}=P^{n} \circ\left(\tilde{r}^{n}, \tilde{s}^{n}\right)^{-1}$ denote the law on $\mathbb{W} \times \mathbb{W}$. For $w=\left(w_{1}, w_{2}\right) \in \mathbb{W} \times \mathbb{W}$, we define the coordinate mappings $\boldsymbol{r}(w)=w_{1}$ and $\boldsymbol{s}(w)=w_{2}$.

Theorem 4. Suppose that $\beta$ and $\gamma$ satisfy Assumptions 4, 5 and 6, and let $\tilde{\mu}$ and $\tilde{\nu}$ be probability measures on $\mathbb{R}_{+}$. Suppose that the sequences $\left(\vartheta^{n}\right)$, $\left(\tilde{\mu}^{n}\right)$ and $\left(\tilde{\nu}^{n}\right)$ satisfy Assumptions 7 and 8 . Then there is a random variable $(\tilde{r}, \tilde{s})$ with values in $\mathbb{W} \times \mathbb{W}$, defined on some probability space $(\Omega, \mathcal{A}, P)$, such that $\left(\tilde{r}_{t}, \tilde{s}_{t}\right)$ is a weak solution of

$$
\begin{aligned}
& d \tilde{r}_{t}=\beta\left(t, \tilde{r}_{t}\right) d t+2 I\left(\tilde{r}_{t}>0\right) d \tilde{W}_{t}, \quad \operatorname{Law}\left(\tilde{r}_{0}, \tilde{s}_{0}\right)=\tilde{\mu} \otimes \tilde{\nu}, \\
& d \tilde{s}_{t}=\gamma\left(t, \tilde{s}_{t}\right) d t+2 I\left(\tilde{s}_{t}>0\right) d \tilde{W}_{t},
\end{aligned}
$$

for some Brownian motion $\left(\tilde{W}_{t}\right)$. Moreover, there is a subsequence $\left(n_{k}\right)$ such that $P^{n_{k}} \circ\left(\tilde{r}^{n_{k}}, \tilde{s}^{n_{k}}\right)^{-1}$ converges weakly towards $P \circ(\tilde{r}, \tilde{s})^{-1}$. If additionally,

$$
\begin{aligned}
\beta(t, x) \leq \gamma(t, x) & \text { for any } x, t \in \mathbb{R}_{+}, \text {and } \\
P^{n}\left[\tilde{r}_{0}^{n} \leq \tilde{s}_{0}^{n}\right]=1 & \text { for any } n \in \mathbb{N},
\end{aligned}
$$

then $P\left[\tilde{r}_{t} \leq \tilde{s}_{t}\right.$ for all $\left.t \geq 0\right]=1$.

Proof. We fix sequences of diffusion coefficients $\left(\vartheta^{n}\right)$ and initial conditions $\left(\tilde{\mu}^{n}\right)$ and $\left(\tilde{\nu}^{n}\right)$ satisfying Assumptions 7 and 8 .

Tightness: We claim that the sequence $\left(\mathbb{P}^{n}\right)_{n \in \mathbb{N}}$ of probability measures on $(\mathbb{W} \times$ $\mathbb{W}, \mathcal{B}(\mathbb{W}) \otimes \mathcal{B}(\mathbb{W}))$ is tight. This can be shown by similar arguments as in $[24,25$, so we only explain briefly how to adapt these arguments to our setting. At first, we observe that a uniform Lyapunov condition holds for the Markov processes $\left(\tilde{r}_{t}^{n}, \tilde{s}_{t}^{n}\right)$ defined by (31). Indeed, these processes solve a local martingale problem w.r.t. the generators

$$
\mathcal{L}_{t}^{n}=\beta(t, \cdot) \partial_{r}+\gamma(t, \cdot) \partial_{s}+2\left(\vartheta^{n}\right)^{2}\left(\partial_{r}^{2}+\partial_{s}^{2}\right)
$$

defined on smooth functions on $\mathbb{R}^{2}$. Let $V(x):=1+|x|^{2}$ for $x \in \mathbb{R}^{2}$. Recall that the drift coefficients in 35 do not depend on $n$ and that they satisfy the linear growth Assumption 6. Moreover, the diffusion coefficients are uniformly bounded by one. It follows that there is a constant $\lambda \in(0, \infty)$, not depending on $n$, such that $\mathcal{L}_{t}^{n} V \leq \lambda V$ for any $n \in \mathbb{N}$. From this one can conclude that for each finite time interval $[0, T]$ and every $\epsilon>0$, there is a compact set $K \subseteq \mathbb{R}^{2}$ such that for any $n \in \mathbb{N}, P\left[\left(\tilde{r}_{t}^{n}, \tilde{s}_{t}^{n}\right) \in K\right.$ for $\left.t \leq T\right] \geq 1-\epsilon$. Moreover, the drift and diffusion coefficients are uniformly bounded on the set $K$. Combining these arguments, we can conclude tightness of the laws on $\mathbb{W} \times \mathbb{W}$. We refer to [24, 25] for a detailled proof in a similar setting. By Prokhorov's Theorem, we can conclude that there is a subsequence $n_{k} \rightarrow \infty$ and a probability measure $\mathbb{P}$ on $\mathbb{W} \times \mathbb{W}$ such that $\mathbb{P}^{n_{k}} \rightarrow \mathbb{P}$ weakly. To simplify notation we will write in the following $n$ instead of $n_{k}$, keeping in mind that we have convergence only along a subsequence.

Identification of the limit: We now characterize the measure $\mathbb{P}$. In principle, we follow well-known strategies for identifying limits of semimartingales, cf. [48, 
30, 16. However, we can not apply those results directly, because the diffusion coefficients in $(32)$ are discontinuous.

We know that $\mathbb{P} \circ\left(\boldsymbol{r}_{0}, \boldsymbol{s}_{0}\right)^{-1}=\mu \otimes \nu$, since $\mathbb{P}^{n} \circ\left(\boldsymbol{r}_{0}, \boldsymbol{s}_{0}\right)^{-1}=\mu^{n} \otimes \nu^{n}$ converges weakly to $\mu \otimes \nu$ by assumption. We define maps $\boldsymbol{M}, \boldsymbol{N}: \mathbb{W} \times \mathbb{W} \rightarrow \mathbb{W}$ by

$$
\boldsymbol{M}_{t}=\boldsymbol{r}_{t}-\boldsymbol{r}_{0}-\int_{0}^{t} \beta\left(u, \boldsymbol{r}_{u}\right) d u \quad \text { and } \quad \boldsymbol{N}_{t}=\boldsymbol{s}_{t}-\boldsymbol{s}_{0}-\int_{0}^{t} \gamma\left(u, \boldsymbol{s}_{u}\right) d u \text {. }
$$

We claim that $\left(\boldsymbol{M}_{t}, \mathcal{F}_{t}, \mathbb{P}\right)$ and $\left(\boldsymbol{N}_{t}, \mathcal{F}_{t}, \mathbb{P}\right)$ are martingales w.r.t. the canonical filtration $\mathcal{F}_{t}=\sigma\left(\left(\boldsymbol{r}_{u}, \boldsymbol{s}_{u}\right)_{0 \leq u \leq t}\right)$. Indeed, the mappings $\boldsymbol{M}$ and $\boldsymbol{N}$ are continuous on $\mathbb{W}$, so by the continuous mapping theorem, $\mathbb{P}^{n} \circ(\boldsymbol{r}, \boldsymbol{s}, \boldsymbol{M}, \boldsymbol{N})^{-1}$ converges weakly to $\mathbb{P} \circ(\boldsymbol{r}, \boldsymbol{s}, \boldsymbol{M}, \boldsymbol{N})^{-1}$. Notice that for each $n \in \mathbb{N},\left(\boldsymbol{M}_{t}, \mathcal{F}_{t}, \mathbb{P}^{n}\right)$ is a martingale. Moreover, for any fixed $t \geq 0$, the family $\left(\boldsymbol{M}_{t}, \mathbb{P}^{n}\right)_{n \in \mathbb{N}}$ is uniformly integrable. Hence $\left(\boldsymbol{M}_{t}, \mathcal{F}_{t}, \mathbb{P}\right)$ is a continuous martingale, cf. [30, Chapter IX, Proposition 1.12]. In particular, the quadratic variation $\left([\boldsymbol{M}]_{t}\right)$ exists $\mathbb{P}$-almost surely. Notice that, by $(31),[\boldsymbol{M}]_{t} \leq 4 t \mathbb{P}^{n}$-almost surely for every $n$. Thus for any $t \geq 0$,

$$
\begin{aligned}
\mathbb{E}\left[\sup _{0 \leq s \leq t}\left|\boldsymbol{M}_{s}\right|^{2}\right] & \leq \liminf _{R \rightarrow \infty} \mathbb{E}\left[\sup _{0 \leq s \leq t}\left|\boldsymbol{M}_{s}\right|^{2} \wedge R\right]=\liminf _{R \rightarrow \infty} \lim _{n \rightarrow \infty} \mathbb{E}^{n}\left[\sup _{0 \leq s \leq t}\left|\boldsymbol{M}_{s}\right|^{2} \wedge R\right] \\
& \leq \liminf _{n \rightarrow \infty} \mathbb{E}^{n}\left[\sup _{0 \leq s \leq t}\left|\boldsymbol{M}_{s}\right|^{2}\right] \leq 4 \liminf _{n \rightarrow \infty} \mathbb{E}^{n}\left[[\boldsymbol{M}]_{t}\right] \leq 16 t,
\end{aligned}
$$

Hence, under $\mathbb{P},\left(\boldsymbol{M}_{t}\right)$ is a square integrable martingale, and thus $\left(\boldsymbol{M}_{t}^{2}-[\boldsymbol{M}]_{t}\right)$ is a martingale, cf. [33, Theorem 21.70]. Similar statements hold for $\left(\boldsymbol{N}_{t}\right)$.

As a next step, we compute the quadratic variations and covariations of $\left(\boldsymbol{M}_{t}\right)$ and $\left(\boldsymbol{N}_{t}\right)$ under $\mathbb{P}$. Here we follow arguments from [44]. Similarly as above, the family $\left(\boldsymbol{M}_{t}^{2}, \mathbb{P}^{n}\right)$ is uniformly integrable for any fixed $t \geq 0$, i.e.,

$$
\lim _{\delta \rightarrow \infty} \sup _{n \in \mathbb{N}} \mathbb{E}^{n}\left[\left|\boldsymbol{M}_{t}\right|^{2} ;\left|\boldsymbol{M}_{t}\right|^{2}>\delta\right]=0 .
$$

Indeed, by Burkholder's inequality, there is a constant $C \in(0, \infty)$ such that

$$
\mathbb{E}^{n}\left[\boldsymbol{M}_{t}^{4}\right] \leq C \mathbb{E}^{n}\left[[\boldsymbol{M}]_{t}^{2}\right] \leq 16 C t^{2} \quad \text { for any } n \in \mathbb{N}
$$

Let $G: \mathbb{W} \rightarrow \mathbb{R}_{+}$be bounded, continuous and non-negative. Equation (36) implies

$$
\lim _{\delta \rightarrow \infty} \sup _{n \in \mathbb{N}} \mathbb{E}^{n}\left[\left|G \boldsymbol{M}_{t}^{2}-G\left(\boldsymbol{M}_{t}^{2} \wedge \delta\right)\right|\right] \leq|G|_{\infty} \liminf _{\delta \rightarrow \infty} \sup _{n \in \mathbb{N}} \mathbb{E}^{n}\left[\boldsymbol{M}_{t}^{2} ; \boldsymbol{M}_{t}^{2}>\delta\right]=0 .
$$

Hence for any such $G$ and any $t \geq 0$,

$$
\begin{aligned}
\mathbb{E}\left[G \boldsymbol{M}_{t}^{2}\right] & =\lim _{\delta \rightarrow \infty} \mathbb{E}\left[G\left(\boldsymbol{M}_{t}^{2} \wedge \delta\right)\right]=\lim _{\delta \rightarrow \infty} \lim _{n \rightarrow \infty} \mathbb{E}^{n}\left[G\left(\boldsymbol{M}_{t}^{2} \wedge \delta\right)\right] \\
& =\lim _{n \rightarrow \infty} \lim _{\delta \rightarrow \infty} \mathbb{E}^{n}\left[G\left(\boldsymbol{M}_{t}^{2} \wedge \delta\right)\right]=\lim _{n \rightarrow \infty} \mathbb{E}^{n}\left[G \boldsymbol{M}_{t}^{2}\right] .
\end{aligned}
$$

We now show that $\left(\boldsymbol{M}_{t}^{2}-4 \int_{0}^{t} I\left(\boldsymbol{r}_{u}>0\right) d u, \mathbb{P}\right)$ is a submartingale. Fix $0 \leq s<t$. Then for any continuous, bounded and $\mathcal{F}_{s}$-measurable function $G: \mathbb{W} \rightarrow \mathbb{R}_{+}$,

$$
\lim _{n \rightarrow \infty} \mathbb{E}^{n}\left[G \int_{s}^{t} 4 \vartheta^{n}\left(\boldsymbol{r}_{u}\right)^{2} d u\right]=\lim _{n \rightarrow \infty} \mathbb{E}^{n}\left[G\left(\boldsymbol{M}_{t}^{2}-\boldsymbol{M}_{s}^{2}\right)\right]=\mathbb{E}\left[G\left(\boldsymbol{M}_{t}^{2}-\boldsymbol{M}_{s}^{2}\right)\right] .
$$


On the other hand, the map $w \mapsto \int_{0}^{\cdot} I\left(w_{s}>\epsilon\right) d s$ from $\mathbb{W}$ to $\mathbb{W}$ is lower semicontinuous for any $\epsilon \geq 0$. Fatou's lemma and the Portemanteau theorem imply

$$
\begin{aligned}
\mathbb{E}\left[G \int_{s}^{t} I\left(\boldsymbol{r}_{u}>0\right) d u\right] & \leq \liminf _{\epsilon \downarrow 0} \mathbb{E}\left[G \int_{s}^{t} I\left(\boldsymbol{r}_{u}>\epsilon\right) d u\right] \\
& \leq \liminf _{\epsilon \downarrow 0} \liminf _{n \rightarrow \infty} \mathbb{E}^{n}\left[G \int_{s}^{t} I\left(\boldsymbol{r}_{u}>\epsilon\right) d u\right] .
\end{aligned}
$$

Notice that for any fixed $\epsilon>0$,

$$
\liminf _{n \rightarrow \infty} \mathbb{E}^{n}\left[G\left(\int_{s}^{t} \vartheta^{n}\left(\boldsymbol{r}_{u}\right)^{2} d u-\int_{s}^{t} I\left(\boldsymbol{r}_{u}>\epsilon\right) d u\right)\right] \geq 0 .
$$

By (38), 39) and 40), we have

$$
\mathbb{E}\left[G\left(\boldsymbol{M}_{t}^{2}-\boldsymbol{M}_{s}^{2}-4 \int_{s}^{t} I\left(\boldsymbol{r}_{u}>0\right) d u\right)\right] \geq 0 .
$$

Invoking a monotone class argument, cf. [43, Theorem 8], we see that $\left(\boldsymbol{M}_{t}^{2}-\right.$ $\left.4 \int_{0}^{t} I\left(\boldsymbol{r}_{s}>0\right) d s, \mathcal{F}_{t}, \mathbb{P}\right)$ is indeed a submartingale. We show that it is also a supermartingale and hence a martingale. By (37), for any function $G$ as above,

$$
\mathbb{E}\left[G\left(\boldsymbol{M}_{t}^{2}-\boldsymbol{M}_{s}^{2}-4(t-s)\right)\right]=\lim _{n \rightarrow \infty} \mathbb{E}^{n}\left[G\left(\boldsymbol{M}_{t}^{2}-\boldsymbol{M}_{s}^{2}-4(t-s)\right)\right] \leq 0
$$

Hence, $\boldsymbol{M}_{t}^{2}-4 t$ is a supermartingale under $\mathbb{P}$. The uniqueness of the Doob-Meyer decomposition [43, Theorem 16] implies that the map $t \mapsto[\boldsymbol{M}]_{t}-4 t$ is $\mathbb{P}$-almost surely decreasing. Observe that $\left(\boldsymbol{r}_{t}, \mathcal{F}_{t}, \mathbb{P}\right)$ is a continuous semimartingale with $[\boldsymbol{r}]=[\boldsymbol{M}]$. Hence the Itô-Tanaka formula implies that $\mathbb{P}$-almost surely,

$$
\int_{0}^{t} I\left(\boldsymbol{r}_{u}=0\right) d[\boldsymbol{M}]_{u}=\int_{0}^{t} I\left(\boldsymbol{r}_{u}=0\right) d[\boldsymbol{r}]_{u}=\int_{-\infty}^{\infty} I(y=0) \ell_{t}^{y}(\boldsymbol{r}) d y=0 .
$$

We conclude that for any $0 \leq s<t$,

$$
[\boldsymbol{M}]_{t}-[\boldsymbol{M}]_{s}=\int_{s}^{t} I\left(\boldsymbol{r}_{u}>0\right) d[\boldsymbol{M}]_{u} \leq 4 \int_{s}^{t} I\left(\boldsymbol{r}_{u}>0\right) d u
$$

and hence for any $\mathcal{F}_{s}$-measurable function $G \in C_{b}(\mathbb{W})$,

$$
\mathbb{E}\left[G\left(\boldsymbol{M}_{t}^{2}-\boldsymbol{M}_{s}^{2}-4 \int_{s}^{t} I\left(\boldsymbol{r}_{u}>0\right) d u\right)\right] \leq 0 .
$$

As above we conclude by a monotone class argument that $\left(\boldsymbol{M}_{t}^{2}-4 \int_{0}^{t} I\left(\boldsymbol{r}_{u}>0\right) d u\right)$ is a supermartingale, and hence a martingale, i.e.,

$$
[\boldsymbol{M}]=4 \int_{0}^{\cdot} I\left(\boldsymbol{r}_{u}>0\right) d u \quad \mathbb{P} \text {-almost surely. }
$$

Similarly, we can show that

$$
[\boldsymbol{N}]=4 \int_{0}^{\cdot} I\left(\boldsymbol{s}_{u}>0\right) d u \quad \mathbb{P} \text {-almost surely. }
$$


Moreover, we claim that

$$
[\boldsymbol{M}, \boldsymbol{N}]=4 \int_{0} I\left(\boldsymbol{r}_{u}>0, \boldsymbol{s}_{u}>0\right) d u \quad \mathbb{P} \text {-almost surely. }
$$

The proof does not involve new arguments, so we just sketch the main steps: With the same arguments as before, one can conclude that

$$
t \mapsto \boldsymbol{M}_{t} \boldsymbol{N}_{t}-4 \int_{0}^{t} I\left(\boldsymbol{r}_{u}>0, \boldsymbol{s}_{u}>0\right) d u
$$

is a submartingale and that the map $t \mapsto \boldsymbol{M}_{t} \boldsymbol{N}_{t}-4 t$ is $\mathbb{P}$-almost surely decreasing. Moreover, by (42), (43), and the Kunita-Watanabe inequality, we see that $\mathbb{P}$-a.s.,

$$
\begin{aligned}
\int_{s}^{t} I\left(\boldsymbol{r}_{u}=0 \text { or } \boldsymbol{s}_{u}=0\right) d[\boldsymbol{M}, \boldsymbol{N}]_{u}=0 \quad \text { for } 0 \leq s \leq t, \quad \text { and thus } \\
\begin{aligned}
{[\boldsymbol{M}, \boldsymbol{N}]_{t}-[\boldsymbol{M}, \boldsymbol{N}]_{s} } & =\int_{s}^{t} I\left(\boldsymbol{r}_{u}>0, \boldsymbol{s}_{u}>0\right) d[\boldsymbol{M}, \boldsymbol{N}]_{u} \\
\leq & 4 \int_{s}^{t} I\left(\boldsymbol{r}_{u}>0, \boldsymbol{s}_{u}>0\right) d u \quad \text { for } 0 \leq s \leq t
\end{aligned}
\end{aligned}
$$

This completes the proof of (44). Invoking a martingale representation theorem, see e.g. [29, Ch. II, Theorem 7.1'], we conclude that there is a probability space $(\Omega, \mathcal{A}, P)$ supporting a Brownian motion $\tilde{W}$, and random variables $(\tilde{r}, \tilde{s})$ such that $P \circ(\tilde{r}, \tilde{s})^{-1}=\mathbb{P} \circ(\boldsymbol{r}, \boldsymbol{s})^{-1}$, and such that $\left(\tilde{r}_{t}, \tilde{s}_{t}, \tilde{W}_{t}\right)$ is a weak solution of 32 .

It remains to show that (33) and (34) imply $P\left[\tilde{r}_{t} \leq \tilde{s}_{t}\right.$ for all $\left.t \geq 0\right]=1$. Applying a comparison theorem [28, Theorem 1] to the approximating diffusions (31) shows that $\mathbb{P}^{n}\left[\boldsymbol{r}_{t} \leq \boldsymbol{s}_{t}\right.$ for all $\left.t \geq 0\right]=1$ for all $n$. The monotonicity carries over to the limit, since $\mathbb{P}^{n} \circ(\boldsymbol{r}, \boldsymbol{s})^{-1}$ converges weakly, along a subsequence, towards $\mathbb{P} \circ(\boldsymbol{r}, \boldsymbol{s})^{-1}$.

3.2. Long time behavior. We now derive bounds for solutions to (29) that are stable for long times. We assume that $t \mapsto \alpha(t, x)$ is non-increasing, so that the stickiness of solutions to 29 is non-decreasing in time.

Assumption 9. The function $\alpha:[0, \infty) \times[0, \infty) \rightarrow \mathbb{R}$ is locally Lipschitz continuous with $\alpha(t, x) \leq \alpha(s, x)$ for any $s \leq t$ and $x \in \mathbb{R}_{+}, \alpha(t, 0)>0$ for any $t \geq 0$, and

$$
\limsup _{r \rightarrow \infty}\left(r^{-1} \alpha(0, r)\right)<0 .
$$

Notice that Assumption 9 implies Assumptions 4,5 and 6 from above.

3.2.1. Invariant measure in the time-homogenous case. We first consider drift coefficients which do not depend on time, i.e., functions of the form $\alpha(t, x)=\alpha(x)$.

Lemma 6. Suppose that Assumption 9 holds true, and $\alpha(t, \cdot)=\alpha$ for a function $\alpha:[0, \infty) \rightarrow \mathbb{R}$. Let $\pi$ be the probability measure on $[0, \infty)$ defined by

$$
\pi(d x)=\frac{1}{Z}\left(\frac{2}{\alpha(0)} \delta_{0}(d x)+\exp \left(\frac{1}{2} \int_{0}^{x} \alpha(y) d y\right) \lambda_{(0, \infty)}(d x)\right)
$$


where $Z=\frac{2}{\alpha(0)}+\int_{0}^{\infty} \exp \left(\frac{1}{2} \int_{0}^{x} \alpha(y) d y\right) d x$. Then $\pi$ is invariant for 29), i.e., if $\left(r_{t}\right)$ is a solution with initial law $\pi$, then $\operatorname{Law}\left(r_{t}\right)=\pi$ for any $t \geq 0$.

Proof. We use an approximation as in (31) with $\beta(t, x)=\alpha(x)$ and a sequence of smooth functions $\vartheta^{n}:[0, \infty) \rightarrow[0,1]$ satisfying Assumption 8 . It is well-known that under our assumptions, for each $n \in \mathbb{N}$, the probability measure $\tilde{\mu}^{n}$ on $\mathbb{R}_{+}$ with distribution function

$$
\tilde{F}^{n}(x)=\frac{\int_{0}^{x} \frac{1}{\vartheta^{n}(y)^{2}} \exp \left(\int_{1 / n}^{y} \frac{\alpha(z)}{2 \vartheta^{n}(z)^{2}} d z\right) d y}{\int_{0}^{\infty} \frac{1}{\vartheta^{n}(y)^{2}} \exp \left(\int_{1 / n}^{y} \frac{\alpha(z)}{2 \vartheta^{n}(z)^{2}} d z\right) d y} \quad x \in[0, \infty),
$$

is an invariant measure for the process $\left(\tilde{r}_{t}^{n}\right)$ defined by (31), see e.g. [37, Chapter 4.4, Theorem 7]. Note in particular that by Assumptions 9 and 8, the occurring integrals are well defined and finite. Let $F$ denote the distribution function of $\pi$. We show that for any $x>0, \tilde{F}^{n}(x) \rightarrow F(x)$ as $n \rightarrow \infty$, which implies that $\tilde{\mu}_{n} \rightarrow \pi$ weakly. Indeed, fix $x \in(0, \infty]$. Then for $n>1 / x$,

$$
\begin{aligned}
& \int_{0}^{x} \frac{1}{\vartheta^{n}(y)^{2}} \exp \left(\int_{1 / n}^{y} \frac{\alpha(z)}{2 \vartheta^{n}(z)^{2}} d z\right) d y \\
= & \int_{1 / n}^{x} \exp \left(\int_{1 / n}^{y} \frac{1}{2} \alpha(z) d z\right) d y+\int_{0}^{1 / n} \frac{1}{\vartheta^{n}(y)^{2}} \exp \left(\int_{1 / n}^{y} \frac{\alpha(z)}{2 \vartheta^{n}(z)^{2}} d z\right) d y .
\end{aligned}
$$

If $C \in(0, \infty)$ is a constant then

$$
\begin{aligned}
& \int_{0}^{1 / n} \frac{1}{\vartheta^{n}(y)^{2}} \exp \left(\int_{1 / n}^{y} \frac{C}{\vartheta^{n}(z)^{2}} d z\right) d y=\lim _{\epsilon \downarrow 0} \int_{\epsilon}^{1 / n} \frac{1}{\vartheta^{n}(y)^{2}} \exp \left(\int_{1 / n}^{y} \frac{C}{\vartheta^{n}(z)^{2}} d z\right) d y \\
& \quad=\lim _{\epsilon \downarrow 0} \frac{1}{C}\left(1-\exp \left(-\int_{\epsilon}^{1 / n} \frac{C}{\vartheta^{n}(z)^{2}} d z\right)\right)=\frac{1}{C} .
\end{aligned}
$$

For $0<y<1 / n$, we have the bounds

$$
\begin{aligned}
\exp \left(\max _{u \in[0,1 / n]} \alpha(u) \int_{1 / n}^{y} \frac{1}{2 \vartheta^{n}(z)^{2}} d z\right) & \leq \exp \left(\int_{1 / n}^{y} \frac{\alpha(z)}{2 \vartheta^{n}(z)^{2}} d z\right) \\
& \leq \exp \left(\min _{u \in[0,1 / n]} \alpha(u) \int_{1 / n}^{y} \frac{1}{2 \vartheta^{n}(z)^{2}} d z\right)
\end{aligned}
$$

Using (47), the continuity of $\alpha$, and (48), we can conclude that as $n \rightarrow \infty$,

$$
\int_{0}^{x} \frac{1}{\vartheta^{n}(y)^{2}} \exp \left(\int_{1 / n}^{y} \frac{\alpha(z)}{2 \vartheta^{n}(z)^{2}} d z\right) d y \rightarrow \int_{0}^{x} \exp \left(\int_{0}^{y} \frac{1}{2} \alpha(z) d z\right) d y+\frac{2}{\alpha(0)} .
$$

Since this also holds for $x=\infty$, we see that $\tilde{F}^{n}(x) \rightarrow F(x)$ for any $x>0$, and hence $\tilde{\mu}_{n} \rightarrow \pi$ weakly. Consequently, by Lemma 4 and Theorem 4 , the laws of the solutions of (31) with initial distributions $\tilde{\mu}^{n}$ converge weakly to the law of the solution of (29) with initial distribution $\pi$. Since the approximating processes are stationary, the limit process is stationary, too. Hence $\pi$ is an invariant measure. 
3.2.2. Long time stability in the time-inhomogeneous case. Let $\left(r_{t}\right)$ be a solution of (29) with an arbitrary but fixed initial distribution $\mu$ on $\mathbb{R}_{+}$. Our aim is to provide bounds on $P\left[r_{t}>0\right]$ and $E\left[r_{t}\right]$ for any fixed $t \geq 0$. To this end we fix a continuous function $a:[0, \infty) \rightarrow \mathbb{R}$ such that

$$
\alpha(0, x) \leq a(x) \text { for any } x \in[0, \infty) \text {, and } \limsup _{r \rightarrow \infty}\left(r^{-1} a(r)\right)<0 .
$$

For example, by Assumption 9, we can always choose $a(x)=\alpha(0, x)$. However, sometimes it can be more convenient to choose the function $a$ in a different way. Following [13, 14] (see also [7, 5, 6, 9]), we define constants $R_{0}, R_{1} \in(0, \infty)$ and a concave function $f: \mathbb{R}_{+} \rightarrow \mathbb{R}_{+}$by

$$
\begin{aligned}
& \text { (50) } R_{0}=\inf \{R \geq 0: a(r) \leq 0 \quad \text { for any } r \geq R\}, \\
& \text { (51) } R_{1}=\inf \left\{R \geq R_{0}: R\left(R-R_{0}\right) a(r) / r \leq-4 \quad \text { for any } r \geq R\right\} \\
& \text { (52) } f(r)=\int_{0}^{r} \varphi(s) g(s) d s, \text { where } \varphi(r)=\exp \left(-\frac{1}{2} \int_{0}^{r} a(s)^{+} d s\right) \text { and } \\
& g(r)=1-\frac{1}{4} \int_{0}^{r \wedge R_{1}} \frac{\Phi(s)}{\varphi(s)} d s / \int_{0}^{R_{1}} \frac{\Phi(s)}{\varphi(s)} d s-\frac{1}{4} \int_{0}^{r \wedge R_{1}} \frac{1}{\varphi(s)} d s / \int_{0}^{R_{1}} \frac{1}{\varphi(s)} d s
\end{aligned}
$$

with $\Phi(r)=\int_{0}^{r} \varphi(s) d s$. The function $f$ is concave, strictly increasing and continuous. Observe that 49 implies that $0<R_{0}<R_{1}<\infty$. We define constants

$$
c=\left(2 \int_{0}^{R_{1}} \frac{\Phi(s)}{\varphi(s)} d s\right)^{-1}, \epsilon=\min \left\{\left(2 \int_{0}^{R_{1}} \frac{1}{\varphi(s)} d s\right)^{-1}, c \Phi\left(R_{1}\right)\right\} .
$$

Notice that $1 / 2 \leq g \leq 1$, and thus $\Phi(r) / 2 \leq f(r) \leq \Phi(r)$. Hence for $0<r<R_{1}$,

$$
2 f^{\prime \prime}(r)+f^{\prime}(r) a(r)^{+} \leq-\epsilon-c \Phi(r) \leq-(\epsilon+c f(r)) .
$$

Lemma 7. Suppose that Assumption 9 holds true. Let $\left(r_{t}\right)$ be a solution of (29), and let $T_{0}=\inf \left\{t \geq 0: r_{t}=0\right\}$. Then for any $t>0$,

$$
\begin{aligned}
E\left[f\left(r_{t}\right) ; t<T_{0}\right] & \leq e^{-c t} E\left[f\left(r_{0}\right)\right], \quad \text { and } \\
P\left[t<T_{0}\right] & \leq \frac{1}{\epsilon} \frac{c}{e^{c t}-1} E\left[f\left(r_{0}\right)\right] .
\end{aligned}
$$

Proof. Notice that the function $f$ can be extended to a concave function on $\mathbb{R}$ by setting $f(x)=x$ for $x<0$. Since the process $\left(r_{t}\right)$ is a continuous semimartingale, we can apply the Itô-Tanaka formula to conclude that almost surely,

$$
d f\left(r_{t}\right)=f^{\prime}\left(r_{t}\right) \alpha\left(t, r_{t}\right) d t+2 f^{\prime \prime}\left(r_{t}\right) I\left(r_{t}>0\right) d t+d M_{t},
$$

where $M_{t}=2 \int_{0}^{t} f^{\prime}\left(r_{s}\right) I\left(r_{s}>0\right) d W_{s}$ is a martingale. By Assumption 9 and 49, $\alpha\left(t, r_{t}\right) \leq \alpha\left(0, r_{t}\right) \leq a\left(r_{t}\right)$. Therefore, for $0<r_{t}<R_{1}$, we can apply (54) to bound the right hand side of (57). On the other hand, for $r_{t} \geq R_{1}$, we have $f^{\prime \prime}\left(r_{t}\right)=0$ and $r_{t}^{-1} \alpha\left(r_{t}\right)<0$. Moreover, by definition of $f$ and $\varphi, f^{\prime}\left(r_{t}\right)=\varphi\left(R_{0}\right) / 2$, and by 
(51), $R_{1}\left(R_{1}-R_{0}\right) \alpha\left(r_{t}\right) / r_{t}^{-1} \leq-4$. Therefore, we can conclude similarly to [14, Proof of Theorem 2.2] that for $r_{t}>R_{1}$,

$$
\begin{aligned}
f^{\prime}\left(r_{t}\right) \alpha\left(t, r_{t}\right) & \leq \varphi\left(R_{0}\right) a\left(r_{t}\right) / 2 \leq-2 \frac{\varphi\left(R_{0}\right)}{R_{1}-R_{0}} \frac{r_{t}}{R_{1}}<-2 \frac{\varphi\left(R_{0}\right)}{R_{1}-R_{0}} \frac{\Phi\left(r_{t}\right)}{\Phi\left(R_{1}\right)} \\
& \leq-\Phi\left(r_{t}\right) / \int_{R_{0}}^{R_{1}} \Phi(s) \varphi(s)^{-1} d s \leq-2 c \Phi\left(r_{t}\right) \\
& \leq-c \Phi\left(R_{1}\right)-c f\left(r_{t}\right) \leq-\left(\epsilon+c f\left(r_{t}\right)\right) .
\end{aligned}
$$

Here we have used that $\int_{R_{0}}^{R_{1}} \Phi(s) \varphi(s)^{-1} d s \geq\left(R_{1}-R_{0}\right) \Phi\left(R_{1}\right) \varphi\left(R_{0}\right)^{-1} / 2$. Combining (57), (54) and (58), we see that almost surely,

$$
d f\left(r_{t}\right) \leq-\left(\epsilon+c f\left(r_{t}\right)\right) d t+d M_{t} \quad \text { for } t<T_{0} .
$$

Using Itô's product rule and (59), we finally obtain

$$
\begin{aligned}
e^{c t} E\left[f\left(r_{t}\right) ; t<T_{0}\right] & \leq E\left[f\left(r_{0}\right)\right]+E\left[e^{c\left(t \wedge T_{0}\right)} f\left(r_{t \wedge T_{0}}\right)-f\left(r_{0}\right)\right] \\
& \leq E\left[f\left(r_{0}\right)\right]-\frac{\epsilon}{c}\left(E\left[e^{c\left(t \wedge T_{0}\right)}\right]-1\right), \quad \text { and } \\
P\left[t<T_{0}\right] & \leq E\left[\frac{e^{c\left(t \wedge T_{0}\right)}-1}{e^{c t}-1}\right] \leq \frac{1}{\epsilon} \frac{c}{e^{c t}-1} E\left[f\left(r_{0}\right)\right] .
\end{aligned}
$$

For $s \in[0, \infty)$, we denote by $\pi_{s}$ the invariant probability measure for the timehomogeneous sticky diffusion with $\operatorname{drift} \alpha(s, \cdot)$ that is given by (46), i.e.,

$$
\pi_{s}(d x) \propto \frac{2}{\alpha(s, 0)} \delta_{0}(d x)+\exp \left(\frac{1}{2} \int_{0}^{x} \alpha(s, y) d y\right) \lambda_{(0, \infty)}(d x)
$$

Theorem 5. Suppose that Assumption 9 holds true, and let $\left(r_{t}\right)$ be a solution of (29) with initial distribution $\mu$ on $\mathbb{R}_{+}$. Then for any $t>0$,

$$
\begin{aligned}
E\left[f\left(r_{t}\right)\right] & \leq e^{-c t} E\left[f\left(r_{0}\right)\right]+\int f d \pi_{0}, \quad E\left[r_{t}\right] \leq 2 \varphi\left(R_{0}\right)^{-1} E\left[f\left(r_{t}\right)\right], \quad \text { and } \\
P\left[r_{t}>0\right] & \leq \frac{1}{\epsilon} \frac{c}{e^{c t}-1} E\left[f\left(r_{0}\right)\right]+\pi_{0}[(0, \infty)] .
\end{aligned}
$$

Proof. Based on the results of Theorem 4, we can construct a filtered probability space $\left(\Omega, \mathcal{A},\left(\mathcal{F}_{t}\right), P\right)$ satisfying the usual conditions and supporting random variables $r, W, \tilde{r}, \tilde{s}, \tilde{W}: \Omega \rightarrow \mathbb{W}$ such that w.r.t. $\left(\Omega, \mathcal{A},\left(\mathcal{F}_{t}\right), P\right)$,

- $(r, W)$ and $(\tilde{r}, \tilde{s}, \tilde{W})$ are independent,

- $\left(r_{t}, W_{t}\right)$ is a weak solution of (29) with initial distribution $\mu$, and

- $\left(\tilde{r}_{t}, \tilde{s}_{t}, \tilde{W}_{t}\right)$ is a weak solution of $(32)$ with $\beta(t, x)=\alpha(t, x), \gamma(t, x)=\alpha(0, x)$, $\tilde{\mu}=\delta_{0}, \tilde{\nu}=\pi_{0}$, and

$$
P\left[\tilde{r}_{t} \leq \tilde{s}_{t} \text { for all } t \geq 0\right]=1 .
$$

Let $T:=\inf \left\{t \geq 0: r_{t}=\tilde{r}_{t}\right\}$ be the first meeting time of $\left(r_{t}\right)$ and $\left(\tilde{r}_{t}\right)$. We define

$$
\bar{r}_{t}:=r_{t} \text { for } t<T, \text { and } \bar{r}_{t}:=\tilde{r}_{t} \text { for } t \geq T \text {. }
$$


Then $\left(\bar{r}_{t}\right)$ solves the martingale problem corresponding to 29 with initial law $\mu$, cf. e.g. [42, Section 3.1]. By Lemma 4, this martingale problem has a unique solution. Hence, we can conclude that the laws of $\bar{r}$ and $r$ on $\mathbb{W}$ coincide. Let $T_{0}=\inf \left\{t \geq 0: r_{t}=0\right\}$. Observe that since $t \mapsto r_{t}$ and $t \mapsto \tilde{r}_{t}$ are continuous with $\tilde{r}_{0}=0 \leq r_{0}$, we have $T \leq T_{0}$. In particular, by Lemma 7) (61), and since $f$ is increasing,

$$
\begin{aligned}
E\left[f\left(r_{t}\right)\right] & =E\left[f\left(\bar{r}_{t}\right)\right]=E\left[f\left(r_{t}\right) ; t<T\right]+E\left[f\left(\tilde{r}_{t}\right) ; t \geq T\right] \\
& \leq E\left[f\left(r_{t}\right) ; t<T_{0}\right]+E\left[f\left(\tilde{s}_{t}\right)\right] \leq e^{-c t} E\left[f\left(r_{0}\right)\right]+\int f d \pi_{0} .
\end{aligned}
$$

Here we have used that by Lemma 6, the process $\left(\tilde{s}_{t}\right)$ is stationary. By $(52),(50)$, and since $g \geq 1 / 2$, we have $f^{\prime} \geq \varphi\left(R_{0}\right) / 2$. Hence the inequality $r \leq 2 \varphi\left(R_{0}\right)^{-1} f(r)$ holds for any $r \geq 0$, and thus, we can conclude that

$$
E\left[r_{t}\right] \leq 2 \varphi\left(R_{0}\right)^{-1} E\left[f\left(r_{t}\right)\right]
$$

Finally, by the second part of Lemma 7, we see that

$$
\begin{aligned}
P\left[r_{t}>0\right] & =P\left[\bar{r}_{t}>0\right]=P\left[r_{t}>0, t<T\right]+P\left[\tilde{r}_{t}>0, t \geq T\right] \\
& \leq P\left[t<T_{0}\right]+P\left[\tilde{s}_{t}>0\right] \leq \frac{1}{\epsilon} \frac{c}{e^{c t}-1} E\left[f\left(r_{0}\right)\right]+\pi_{0}[(0, \infty)]
\end{aligned}
$$

By applying Theorem 5 on the time intervals $[s, t]$ and $[0, s]$, we obtain:

Corollary 1. Suppose that Assumption 9 holds true, and let $\left(r_{t}\right)$ be a solution of 29. Then for any $0 \leq s<t$,

$$
\begin{aligned}
E\left[f\left(r_{t}\right)\right] & \leq e^{-c t} E\left[f\left(r_{0}\right)\right]+e^{-c(t-s)} \int f d \pi_{0}+\int f d \pi_{s}, \quad \text { and } \\
P\left[r_{t}>0\right] & \leq \frac{1}{\epsilon} \frac{c}{e^{c(t-s)}-1}\left(e^{-c s} E\left[f\left(r_{0}\right)\right]+\int f d \pi_{0}\right)+\pi_{s}[(0, \infty)] .
\end{aligned}
$$

where $f, c$ and $\epsilon$ are defined as above. Furthermore,

$$
\begin{aligned}
& E\left[f_{s}\left(r_{t}\right)\right] \leq \frac{2}{\varphi\left(R_{0}\right)} e^{-c_{s}(t-s)}\left(e^{-c s} E\left[f\left(r_{0}\right)\right]+\int f d \pi_{0}\right)+\int f_{s} d \pi_{s}, \quad \text { and } \\
& P\left[r_{t}>0\right] \leq \frac{2}{\varphi\left(R_{0}\right) \epsilon_{s}} \frac{c_{s}}{e^{c_{s}(t-s)}-1}\left(e^{-c s} E\left[f\left(r_{0}\right)\right]+\int f d \pi_{0}\right)+\pi_{s}[(0, \infty)],
\end{aligned}
$$

where $f_{s}, c_{s}$ and $\epsilon_{s}$ are defined by (52), (53) and (46) with a replaced by $\alpha(s, \cdot)$.

Proof. Fix $s \in[0, \infty)$. Then the process $\left(r_{s+t}\right)_{t \geq 0}$ solves $(29)$ with drift coefficient $\alpha_{s}(t, x)=\alpha(s+t, x)$ and initial distribution $P \circ r_{s}^{-1}$. Since $\alpha_{s}(t, x) \leq \alpha(s, x) \leq a(x)$ for any $t, x \geq 0$, we can apply Theorem 5 either with $a, f, c$ and $\epsilon$ as above, or with 
$a, f, c$ and $\epsilon$ replaced by $\alpha(s, \cdot), f_{s}, c_{s}$ and $\epsilon_{s}$. For $t>s$ we obtain

$$
\begin{aligned}
& E\left[f\left(r_{t}\right)\right] \leq e^{-c(t-s)} E\left[f\left(r_{s}\right)\right]+\int f d \pi_{s}, \\
& P\left[r_{t}>0\right] \leq \frac{1}{\epsilon} \frac{c}{e^{c(t-s)}-1} E\left[f\left(r_{s}\right)\right]+\pi_{s}[(0, \infty)], \\
& E\left[f_{s}\left(r_{t}\right)\right] \leq e^{-c_{s}(t-s)} E\left[f_{s}\left(r_{s}\right)\right]+\int f_{s} d \pi_{s}, \\
& P\left[r_{t}>0\right] \leq \frac{1}{\epsilon_{s}} \frac{c_{s}}{e^{c_{s}(t-s)}-1} E\left[f_{s}\left(r_{s}\right)\right]+\pi_{s}[(0, \infty)] .
\end{aligned}
$$

Noting that $f_{s}\left(r_{s}\right) \leq r_{s}$, the assertion follows by applying Theorem 5 once more.

\section{Coupling construction and proofs of the main Results}

In this section, we prove our main theorems. First of all, we construct the sticky coupling $\left(X_{t}, Y_{t}\right)$ of solutions to (1) and (2) respectively, advertised in Theorem 1 . The coupling is obtained as a weak limit of Markovian couplings $\left(X_{t}^{\delta}, Y_{t}^{\delta}\right), \delta>0$. The couplings $\left(X_{t}^{\delta}, Y_{t}^{\delta}\right)$ are reflection couplings for $\left|X_{t}^{\delta}-Y_{t}^{\delta}\right| \geq \delta$ and synchronous couplings for $\left|X_{t}^{\delta}-Y_{t}^{\delta}\right|=0$. Inbetween there is an interpolation between the two types of couplings. We argue that the family of couplings is tight and thus there is a subsequence converging to a coupling $\left(X_{t}, Y_{t}\right)_{t \geq 0}$. It is then argued that this limiting coupling is sticky and shares the properties stated in Theorem 1 .

We now define the couplings $\left(X_{t}^{\delta}, Y_{t}^{\delta}\right)$ rigorously. The technical realization follows [14]. We introduce Lipschitz functions $\mathrm{rc}^{\delta}, \mathrm{sc}^{\delta}: \mathbb{R}_{+} \rightarrow[0,1]$ such that $\mathrm{rc}^{\delta}(0)=0, \mathrm{rc}^{\delta}(r)>0$ for $0<r<\delta, \mathrm{rc}^{\delta}(r)=1$ for $r \geq \delta$, and

$$
\mathrm{rc}^{\delta}(r)^{2}+\mathrm{sc}^{\delta}(r)^{2}=1 \text { for any } r \geq 0 .
$$

Let $\left(B_{t}^{1}\right)$ and $\left(B_{t}^{2}\right)$ be independent $d$-dimensional Brownian motions, and let $u \in \mathbb{R}^{d}$ be some arbitrary unit vector. We define the coupling $\left(X_{t}^{\delta}, Y_{t}^{\delta}\right)$ for (1) and (2) as a diffusion process in $\mathbb{R}^{2 d}$ satisfying the stochastic differential equation

$$
\begin{aligned}
d X_{t}^{\delta} & =b\left(t, X_{t}^{\delta}\right) d t+ & \operatorname{rc}^{\delta}\left(\tilde{r}_{t}^{\delta}\right) d B_{t}^{1}+\operatorname{sc}^{\delta}\left(\tilde{r}_{t}^{\delta}\right) d B_{t}^{2} \\
d Y_{t}^{\delta} & =\tilde{b}\left(t, Y_{t}^{\delta}\right) d t+\operatorname{rc}^{\delta}\left(\tilde{r}_{t}^{\delta}\right) & \left(\operatorname{Id}_{\mathbb{R}^{d}}-2 e_{t}^{\delta}\left\langle e_{t}^{\delta}, \cdot\right\rangle\right) d B_{t}^{1}+\operatorname{sc}^{\delta}\left(\tilde{r}_{t}^{\delta}\right) d B_{t}^{2}
\end{aligned}
$$

with initial condition $\left(X_{0}^{\delta}, Y_{0}^{\delta}\right)=(x, y)$. Here $Z_{t}^{\delta}=X_{t}^{\delta}-Y_{t}^{\delta}, \tilde{r}_{t}^{\delta}=\left|Z_{t}^{\delta}\right|, e_{t}^{\delta}=Z_{t}^{\delta} / \tilde{r}_{t}^{\delta}$ if $\tilde{r}_{t}^{\delta} \neq 0$, and $e_{t}^{\delta}=u$ if $\tilde{r}_{t}^{\delta}=0$. Since $\operatorname{rc}^{\delta}(0)=0$, the arbitrary value $u$ is not relevant for the dynamics. The process $\left(X_{t}^{\delta}, Y_{t}^{\delta}\right)$ can be realized as a standard diffusion process in $\mathbb{R}^{2 d}$ with locally Lipschitz coefficients. Moreover, Assumptions 1 and 2 imply the non-explosiveness of the process. Using Lévy's characterization of Brownian motion and (63), one can check that $\left(X_{t}^{\delta}, Y_{t}^{\delta}\right)$ is indeed a coupling of solutions to Equations (1) and (2). Notice that the process $W_{t}^{\delta}=\int_{0}^{t}\left\langle e_{s}^{\delta}, d B_{s}^{1}\right\rangle$ is a one-dimensional Brownian motion. 
Lemma 8. Suppose that Assumptions 1 and 2 are satisfied. Then, almost surely,

$$
\begin{aligned}
d \tilde{r}_{t}^{\delta} & =\left\langle e_{t}^{\delta}, b\left(t, X_{t}^{\delta}\right)-\tilde{b}\left(t, Y_{t}^{\delta}\right)\right\rangle d t+2 \operatorname{rc}^{\delta}\left(\tilde{r}_{t}^{\delta}\right) d W_{t}^{\delta} \\
& \leq\left(M+\kappa\left(\tilde{r}_{t}^{\delta}\right) \tilde{r}_{t}^{\delta}\right) d t+2 \operatorname{rc}^{\delta}\left(\tilde{r}_{t}^{\delta}\right) d W_{t}^{\delta} .
\end{aligned}
$$

Proof. By 64 and 65,

$$
d\left(\tilde{r}_{t}^{\delta}\right)^{2}=2\left\langle Z_{t}^{\delta}, b\left(t, X_{t}^{\delta}\right)-\tilde{b}\left(t, Y_{t}^{\delta}\right)\right\rangle d t+4 \operatorname{rc}^{\delta}\left(\tilde{r}_{t}^{\delta}\right)^{2} d t+4 \operatorname{rc}^{\delta}\left(\tilde{r}_{t}^{\delta}\right)\left\langle Z_{t}^{\delta}, e_{t}^{\delta}\right\rangle d W_{t}^{\delta}
$$

For $\epsilon>0$, we define a $C^{2}$ approximation of the square root by

$$
S_{\epsilon}(r)=-(1 / 8) \epsilon^{-3 / 2} r^{2}+(3 / 4) \epsilon^{-1 / 2} r+(3 / 8) \epsilon^{1 / 2} \quad \text { for } r<\epsilon,
$$

$S_{\epsilon}(r)=\sqrt{r}$ for $r \geq \epsilon$. By Itô's formula,

$$
\begin{aligned}
d S_{\epsilon}\left(\left(\tilde{r}_{t}^{\delta}\right)^{2}\right) & =2 S_{\epsilon}^{\prime}\left(\left(\tilde{r}_{t}^{\delta}\right)^{2}\right)\left\langle Z_{t}^{\delta}, b\left(t, X_{t}^{\delta}\right)-\tilde{b}\left(t, Y_{t}^{\delta}\right)\right\rangle d t+4 S_{\epsilon}^{\prime}\left(\left(\tilde{r}_{t}^{\delta}\right)^{2}\right) \operatorname{rc}^{\delta}\left(\tilde{r}_{t}^{\delta}\right)^{2} d t \\
& +8 S_{\epsilon}^{\prime \prime}\left(\left(\tilde{r}_{t}^{\delta}\right)^{2}\right) \operatorname{rc}^{\delta}\left(\tilde{r}_{t}^{\delta}\right)^{2}\left(\tilde{r}_{t}^{\delta}\right)^{2} d t+4 S_{\epsilon}^{\prime}\left(\left(\tilde{r}_{t}^{\delta}\right)^{2}\right) \operatorname{rc}^{\delta}\left(\tilde{r}_{t}^{\delta}\right) \tilde{r}_{t}^{\delta} d W_{t}^{\delta} .
\end{aligned}
$$

We can now pass to the limit $\epsilon \downarrow 0$ to obtain (66). Notice that $\sup _{0 \leq r \leq \epsilon}\left|S_{\epsilon}^{\prime}(r)\right| \lesssim$ $\epsilon^{-1 / 2}, \sup _{0 \leq r \leq \epsilon}\left|S_{\epsilon}^{\prime \prime}(r)\right| \lesssim \epsilon^{-3 / 2}$ and that $\mathrm{rc}^{\delta}$ is Lipschitz with $\operatorname{rc}^{\delta}(0)=0$. Hence, one can use Lebesgue's dominated convergence theorem for the convergence of the first three integrals. Moreover, the stochastic integral converges almost surely, along a subsequence, to $\int_{0}^{t} 2 \operatorname{rc}^{\delta}\left(\tilde{r}_{s}^{\delta}\right) d W_{s}^{\delta}$. Finally, by Assumptions 1 and 2 .

$$
\begin{aligned}
\left\langle Z_{t}^{\delta}, b\left(t, X_{t}^{\delta}\right)-\tilde{b}\left(t, Y_{t}^{\delta}\right)\right\rangle & \leq\left\langle Z_{t}^{\delta}, b\left(t, X_{t}^{\delta}\right)-b\left(t, Y_{t}^{\delta}\right)+b\left(t, Y_{t}^{\delta}\right)-\tilde{b}\left(t, Y_{t}^{\delta}\right)\right\rangle \\
& \leq M \tilde{r}_{t}^{\delta}+\kappa\left(\tilde{r}_{t}^{\delta}\right)\left(\tilde{r}_{t}^{\delta}\right)^{2} .
\end{aligned}
$$

In order to control the distance of $X_{t}^{\delta}$ and $Y_{t}^{\delta}$, we introduce a one-dimensional process $\left(r_{t}^{\delta}\right)$ that is defined as the unique and strong solution to the equation

$$
d r_{t}^{\delta}=\left(M+\kappa\left(r_{t}^{\delta}\right) \cdot r_{t}^{\delta}\right) d t+2 \mathrm{rc}^{\delta}\left(r_{t}^{\delta}\right) d W_{t}^{\delta}, \quad r_{0}^{\delta}=\tilde{r}_{0}^{\delta},
$$

with $\left(\tilde{r}_{t}^{\delta}\right)$ and $\left(W_{t}^{\delta}\right)$ as above.

Lemma 9. We have $\left|X_{t}^{\delta}-Y_{t}^{\delta}\right|=\tilde{r}_{t}^{\delta} \leq r_{t}^{\delta}$, almost surely for all $t \geq 0$.

Proof. The processes $\left(\tilde{r}_{t}^{\delta}\right)$ and $\left(r_{t}^{\delta}\right)$ are driven by the same noise, start at the same position, and, by (67), the drift of $\left(\tilde{r}_{t}^{\delta}\right)$ is smaller or equal to the one of $\left(r_{t}^{\delta}\right)$. Therefore, the assertion follows by Ikeda-Watanabe's comparison theorem for onedimensional diffusions, cf. [28, Theorem 1.1].

Proof of Theorem 1. We consider the diffusion $U_{t}^{\delta}:=\left(X_{t}^{\delta}, Y_{t}^{\delta}, r_{t}^{\delta}\right)$ on $\mathbb{R}^{2 d+1}$. Let $\mathbb{P}^{\delta}$ denote the law of $U^{\delta}$ on the space $C\left(\mathbb{R}_{+}, \mathbb{R}^{2 d+1}\right)$. We define $\boldsymbol{X}, \boldsymbol{Y}: C\left(\mathbb{R}_{+}, \mathbb{R}^{2 d+1}\right) \rightarrow$ $C\left(\mathbb{R}_{+}, \mathbb{R}^{d}\right)$ and $\boldsymbol{r}: C\left(\mathbb{R}_{+}, \mathbb{R}^{2 d+1}\right) \rightarrow C\left(\mathbb{R}_{+}, \mathbb{R}\right)$ as the canonical projections onto the first $d$, the second $d$, and the last coordinate.

Notice that in each of the equations (64), (65) and $(69)$, the drift coefficients do not depend on $\delta$ and the diffusion coefficients are uniformly bounded. Moreover, Assumptions 1 and 2 imply that, similarly as in the proof of Theorem 4 , the diffusions $\left(U_{t}^{\delta}\right)$ satisfy uniformly a Lyapunov non-explosion criterion, and the drift 
coefficients are uniformly bounded on compact sets. Therefore, the family $\left(\mathbb{P}^{\delta}\right)$ is tight, cf. [24, 25]. In particular, there is a sequence $\delta_{n} \downarrow 0$ such that $\left(\mathbb{P}^{\delta_{n}}\right)$ converges towards a measure $\mathbb{P}$ on $\mathbb{C}\left(\mathbb{R}_{+}, \mathbb{R}^{2 d+1}\right)$. For each $\delta>0,\left(X_{t}^{\delta}\right)$ and $\left(Y_{t}^{\delta}\right)$ are solutions to (1) and (2) respectively. Since those solutions are unique in law, we know that $\mathbb{P}^{\delta} \circ\left(X^{\delta}\right)^{-1}=\mathbb{P} \circ \boldsymbol{X}^{-1}$ and $\mathbb{P}^{\delta} \circ\left(Y^{\delta}\right)^{-1}=\mathbb{P} \circ \boldsymbol{Y}^{-1}$ for any $\delta>0$. Hence, $\mathbb{P} \circ(\boldsymbol{X}, \boldsymbol{Y})^{-1}$ is a coupling of (1) and (2). Moreover, Lemma 4 and the proof of Theorem 4 reveal that, after extending the underlying probability space, there is a Brownian motion $\left(\tilde{W}_{t}\right)$ such that $\left(r_{t}, \tilde{W}_{t}\right)$ is a solution of $(3)$. The statement from Lemma 9 carries over to the limiting processes, since such inequalities are preserved under weak convergence, and thus (4) holds. The inequality (8) is implied by Theorem 5 setting $\alpha(t, x)=a(x)=M+\kappa(x) \cdot x$.

Proof of Lemma 1. By (6), $\pi[(0, \infty)]=\frac{\alpha}{1+\alpha}$ with

$$
\alpha:=\frac{M}{2} \int_{0}^{\infty} \exp \left(\frac{1}{2} \int_{0}^{x}(M+\kappa(y) y) d y\right) d x .
$$

In order to provide upper bounds on $\alpha$, we decompose $\alpha=M(a+b) / 2$ with

$$
\begin{aligned}
a & =\int_{\mathcal{R}}^{\infty} \exp \left(\frac{1}{2} \int_{0}^{x}(M+\kappa(y) y) d y\right) d x \quad \text { and } \\
b & =\int_{0}^{\mathcal{R}} \exp \left(\frac{1}{2} \int_{0}^{x}(M+\kappa(y) y) d y\right) d x
\end{aligned}
$$

By Condition (13), we have

$$
\begin{aligned}
\frac{1}{2} \int_{0}^{x}(M+\kappa(y) y) d y & =\frac{1}{2} \int_{0}^{\mathcal{R}}(M+L y) d y+\frac{1}{2} \int_{\mathcal{R}}^{x}(M-K y) d y \\
& =M x / 2-K x^{2} / 4+(L+K) \mathcal{R}^{2} / 4 \\
& =-K(x-M / K)^{2} / 4+M^{2} /(4 K)+(L+K) \mathcal{R}^{2} / 4
\end{aligned}
$$

for $x \geq \mathcal{R}$ and

$$
\frac{1}{2} \int_{0}^{x}(M+\kappa(y) y) d y=\frac{1}{2} \int_{0}^{x}(M+L y) d y=M x / 2+L x^{2} / 4
$$

for $x \leq \mathcal{R}$. We obtain

$$
\begin{aligned}
a & =\exp \left(M^{2} /(4 K)+(L+K) \mathcal{R}^{2} / 4\right) \int_{\mathcal{R}}^{\infty} \exp \left(-K(x-M / K)^{2} / 4\right) d x \\
& =\frac{\sqrt{2}}{\sqrt{K}} \exp \left(M^{2} /(4 K)+(L+K) \mathcal{R}^{2} / 4\right) \int_{(\mathcal{R}-M / K) \sqrt{K / 2}}^{\infty} \exp \left(-z^{2} / 2\right) d z \text { and } \\
b & =\int_{0}^{\mathcal{R}} \exp \left(M x / 2+L x^{2} / 4\right) d x
\end{aligned}
$$


and give upper bounds for these quantities:

$$
\begin{aligned}
(70) b & \leq \mathcal{R} \exp \left(M \mathcal{R} / 2+L \mathcal{R}^{2} / 4\right) \\
(71) b & =\exp \left(M \mathcal{R} / 2+L \mathcal{R}^{2} / 4\right) \int_{0}^{\mathcal{R}} \exp \left(M(\mathcal{R}-x) / 2-L\left(\mathcal{R}^{2}-x^{2}\right) / 4\right) d x \\
& =\exp \left(M \mathcal{R} / 2+L \mathcal{R}^{2} / 4\right) \int_{0}^{\mathcal{R}} \exp (-M y / 2-L y(2 \mathcal{R}-y) / 4) d y \\
& \leq \exp \left(M \mathcal{R} / 2+L \mathcal{R}^{2} / 4\right) \int_{0}^{\mathcal{R}} \exp (-M y / 2-L \mathcal{R} y / 4) d y \\
& \leq \frac{1}{M / 2+L \mathcal{R} / 4} \exp \left(M \mathcal{R} / 2+L \mathcal{R}^{2} / 4\right)
\end{aligned}
$$

Combining (70) and (71), we conclude that

$$
b \leq \frac{4 \mathcal{R}}{\max \left(4,2 M \mathcal{R}+L \mathcal{R}^{2}\right)} \exp \left(M \mathcal{R} / 2+L \mathcal{R}^{2} / 4\right) \text {. }
$$

We use the bound $\int_{0}^{\infty} e^{-z^{2} / 2} d z \leq \sqrt{2 \pi}$ to conclude that

$$
\begin{aligned}
a & \leq 2 \sqrt{\pi / K} \exp \left(M^{2} /(4 K)+(L+K) \mathcal{R}^{2} / 4\right) \\
& =2 \sqrt{\pi / K} \exp \left(K(R-M / K)^{2} / 4\right) \exp \left(M \mathcal{R} / 2+L \mathcal{R}^{2} / 4\right) \\
& \leq 2 \sqrt{\frac{\pi e}{K}} \exp \left(M \mathcal{R} / 2+L \mathcal{R}^{2} / 4\right) \quad \text { for } K(R-M / K)^{2} \leq 2
\end{aligned}
$$

On the other hand, $\int_{y}^{\infty} e^{-z^{2} / 2} d z \leq e^{-y^{2} / 2} / y$ for any $y>0$ and thus

$$
\begin{aligned}
(74) a & \leq \frac{2}{K} \frac{1}{\mathcal{R}-M / K} \exp \left(\left(-K(\mathcal{R}-M / K)^{2}+M^{2} / K+(L+K) \mathcal{R}^{2}\right) / 4\right) \\
& =\frac{2}{\sqrt{K}} \frac{1}{\sqrt{K(R-M / K)^{2}}} \exp \left(M \mathcal{R} / 2+L \mathcal{R}^{2} / 4\right) \\
& \leq \frac{\sqrt{2}}{\sqrt{K}} \exp \left(M \mathcal{R} / 2+L \mathcal{R}^{2} / 4\right)
\end{aligned}
$$

provided $\mathcal{R} \geq M / K$ and $K(R-M / K)^{2} \geq 2$. Combining (72), (73) and (74), we obtain in the case $\mathcal{R} \geq M / K$ the bound

$$
\begin{aligned}
\alpha & =M(a+b) / 2 \\
& \leq\left(\pi^{1 / 2} e^{1 / 2} K^{-1 / 2}+2 \mathcal{R} \max \left(4, L \mathcal{R}^{2}+2 M \mathcal{R}\right)^{-1}\right) M \exp \left(M \mathcal{R} / 2+L \mathcal{R}^{2} / 4\right)
\end{aligned}
$$

In the case $\mathcal{R} \leq M / K, 72$ implies

$$
b \leq \frac{4 \mathcal{R}}{\max \left(4,2 M \mathcal{R}+L \mathcal{R}^{2}\right)} \exp \left(M^{2} /(4 K)+(L+K) \mathcal{R}^{2} / 4\right) .
$$

Combining 75 and $(73)$, we can conclude for $\mathcal{R} \leq M / K$ the bound

$$
\alpha \leq\left(\sqrt{\frac{\pi}{K}}+\frac{2 \mathcal{R}}{\max \left(4,2 M \mathcal{R}+L \mathcal{R}^{2}\right)}\right) M \exp \left(\frac{M^{2}}{4 K}+\frac{L+K}{4} \mathcal{R}^{2}\right) .
$$


Proof of Theorem 3. The proof is similar to the proof of Theorem 1. We fix $x_{0}, y_{0} \in$ $\mathbb{R}^{d}$ and corresponding drifts $b(t, x)=b^{x_{0}}(t, x)$ and $\tilde{b}(t, x)=b^{y_{0}}(t, x)$ as in $(22)$ and (23) respectively. Moreover, we choose $\tau_{0} \in(0, \infty)$ such that 26$)$ holds for $|\tau| \leq \tau_{0}$. Since $\vartheta$ is Lipschitz, we can conclude by $(26)$ that for any $x \in \mathbb{R}^{d}$,

$$
\begin{aligned}
|b(t, x)-\tilde{b}(t, x)| & =|\tau| \cdot\left|\int \vartheta(x, y) \mu_{t}^{x_{0}}(d y)-\int \vartheta(x, y) \mu_{t}^{y_{0}}(d y)\right| \\
& \leq|\tau| L \mathcal{W}^{1}\left(\mu_{t}^{x_{0}}, \mu_{t}^{y_{0}}\right) \leq L A e^{-\lambda t}\left|x_{0}-y_{0}\right|
\end{aligned}
$$

where $L$ is the corresponding Lipschitz constant. We can now repeat the procedure leading to the proof of Theorem 1, replacing $M$ by $|\tau| L A e^{-\lambda t}\left|x_{0}-y_{0}\right|$. In particular, we can conclude that there is a coupling $\left(X_{t}, Y_{t}\right)$ of 24 and 25 and a solution $\left(r_{t}, W_{t}\right)$ of (29) with $r_{0}=\left|x_{0}-y_{0}\right|$ and drift

$$
\alpha(t, x)=|\tau| L A e^{-\lambda t}\left|x_{0}-y_{0}\right|+\kappa(x) x
$$

such that $\left|X_{t}-Y_{t}\right| \leq r_{t}$. Notice that Assumption 3 implies Assumption 9 for the drift $\alpha$. We now want to apply Corollary 1. First, we fix the function $a$ in $(49)$ as $a(\cdot):=\alpha(0, \cdot)$. Applying Corollary 1 now yields that for any $0 \leq s<t$,

$$
\left\|\mu_{t}^{x_{0}}-\mu_{t}^{y_{0}}\right\|_{T V} \leq \frac{1}{\epsilon} \frac{c}{e^{c(t-s)}-1}\left(e^{-c s} f\left(\left|x_{0}-y_{0}\right|\right)+\int f d \pi_{0}\right)+\pi_{s}[(0, \infty)] .
$$

By 60, Assumption 9, and since $f(r) \leq r$, we have $f\left(\left|x_{0}-y_{0}\right|\right) \leq\left|x_{0}-y_{0}\right|$ and $\int f d \pi_{0}<\infty$. Moreover, by (60), (76) and Assumption 9 .

$$
\pi_{s}[(0, \infty)] \leq \frac{1}{2} \alpha(s, 0) \int_{0}^{\infty} \exp \left(\frac{1}{2} \int_{0}^{x} \alpha(s, y) d y\right) d x \leq C e^{-\lambda s}
$$

where $C:=\frac{1}{2}|\tau| L A \int_{0}^{\infty} \exp \left(\frac{1}{2} \int_{0}^{x} \alpha(s, y) d y\right) d x$ is a finite constant. Thus, there is a constant $A \in(0, \infty)$ such that

$$
\left\|\mu_{t}^{x_{0}}-\mu_{t}^{y_{0}}\right\|_{T V} \leq \frac{A}{e^{c(t-s)}-1}+C e^{-\lambda s}=e^{-c(t-s)} \frac{A}{1-e^{-c(t-s)}}+C e^{-\lambda s}
$$

for any $0 \leq s<t$. We can now set $s=t / 2$ and use the boundedness of $\|\cdot\|_{T V}$ to see that there is a constant $B \in(0, \infty)$ such that

$$
\left\|\mu_{t}^{x_{0}}-\mu_{t}^{y_{0}}\right\|_{T V} \leq B \exp (-\min (c, \lambda) t / 2) \quad \text { for all } t \geq 0 .
$$

It should be stressed, that the constants $B$ and $c$ depend on the initial conditions.

\section{Appendix A. Computations for Example 2}

In this appendix we prove lower bounds on the total variation distance between the probability measures $\nu(d x)=Z_{f}^{-1} f(x) d x$ and $\mu(d x)=Z_{g}^{-1} g(x) d x$ on $\mathbb{R}^{1}$ that have been considered in Example 2. Noticing that by symmetry of $f$,

$$
\begin{aligned}
Z_{g} & =\int_{-\infty}^{\infty} g(x) d x=\int_{-\infty}^{\infty} e^{m x} f(x) d x=\int_{0}^{\infty}\left(e^{m x}+e^{-m x}\right) f(x) d x \\
& \geq 2 \int_{0}^{\infty} f(x) d x=\int_{-\infty}^{\infty} f(x) d x=Z_{f}, \quad \text { we obtain }
\end{aligned}
$$




$$
\begin{aligned}
& \|\mu-\nu\|_{T V}=\int_{\mathbb{R}}(1-d \mu / d \nu)^{+} d \nu=\int_{\mathbb{R}}\left(1-e^{m x} Z_{f} / Z_{g}\right)^{+} \nu(d x) \\
& \geq \quad \int_{-\infty}^{0}\left(1-e^{m x}\right) \nu(d x)=\int_{0}^{\infty}\left(1-e^{-m x}\right) \nu(d x) \\
& =\quad \nu[(0, R)] \int_{0}^{R}\left(1-e^{-m x}\right) d x / R \\
& \quad+\nu[(R, \infty)] \int_{R}^{\infty}\left(1-e^{-m x}\right) e^{-k(x-R)^{2} / 2} d x / \int_{R}^{\infty} e^{-k(x-R)^{2} / 2} d x \\
& =\quad \nu[(0, R)]\left(m R-1+e^{-m R}\right) /(m R) \\
& \quad+\nu[(R, \infty)] \int_{0}^{\infty}\left(1-e^{-m(R+t)}\right) e^{-k t^{2} / 2} d t / \int_{0}^{\infty} e^{-k t^{2} / 2} d t .
\end{aligned}
$$

Using that $\left(e^{-x}-1+x\right) / x \leq 1-e^{-x}$ for any $x>0$, we obtain the lower bound

$$
\|\mu-\nu\|_{T V} \geq\left(e^{-m R}-1+m R\right) /(m R) .
$$

We now derive an improved bound for small $k$. Suppose that $R \sqrt{k} \leq 1$. Then

$$
\nu[(R, \infty)] / \nu[(0, R)]=\int_{0}^{\infty} e^{-k t^{2} / 2} d t / R=\sqrt{\pi /(2 k)} R^{-1}
$$

implies $\nu[(R, \infty)]=\frac{1}{2}(1+R \sqrt{2 k / \pi})^{-1} \geq \frac{1}{4}$. Hence by (77),

$$
\begin{aligned}
\|\mu-\nu\|_{T V} & \geq \frac{1}{4} \int_{0}^{\infty}\left(1-e^{-m(R+t)}\right) e^{-k t^{2} / 2} d t / \int_{0}^{\infty} e^{-k t^{2} / 2} d t \\
& =\frac{1}{4}\left(1-e^{-m R+m^{2} /(2 k)}\left(1-\sqrt{2 / \pi} \int_{0}^{m / \sqrt{k}} e^{-s^{2} / 2} d s\right)\right) \\
& \geq \frac{1}{4}\left(1-e^{-m R+m^{2} /(2 k)}+\sqrt{2 /(\pi k)} m e^{-m R}\right) .
\end{aligned}
$$

\section{REFERENCES}

[1] Madjid Amir. Sticky Brownian motion as the strong limit of a sequence of random walks. Stochastic Process. Appl., 39(2):221-237, 1991.

[2] Richard F. Bass. A stochastic differential equation with a sticky point. Electron. J. Probab., 19:no. 32, 22, 2014.

[3] V. I. Bogachev, A. I. Kirillov, and S. V. Shaposhnikov. The Kantorovich and variation distances between invariant measures of diffusions and nonlinear stationary Fokker-PlanckKolmogorov equations. Math. Notes, 96(5-6):855-863, 2014.

[4] V. I. Bogachev, M. Röckner, and S. V. Shaposhnikov. Distances between transition probabilities of diffusions and applications to nonlinear Fokker-Planck-Kolmogorov equations. $J$. Funct. Anal., 271(5):1262-1300, 2016.

[5] M. F. Chen and F.-Y. Wang. Estimation of the first eigenvalue of second order elliptic operators. J. Funct. Anal., 131(2):345-363, 1995.

[6] M.-F. Chen and F.-Y. Wang. Estimation of spectral gap for elliptic operators. Trans. Amer. Math. Soc., 349(3):1239-1267, 1997.

[7] $\mathrm{Mu} \mathrm{Fa}$ Chen and Shao Fu Li. Coupling methods for multidimensional diffusion processes. Ann. Probab., 17(1):151-177, 1989. 
[8] R. Chitashvili. On the nonexistence of a strong solution in the boundary problem for a sticky Brownian motion. Proc. A. Razmadze Math. Inst., 115:17-31, 1997.

[9] G. Da Prato, A. Debussche, and L. Tubaro. Coupling for some partial differential equations driven by white noise. Stochastic Process. Appl., 115(8):1384-1407, 2005.

[10] Anirban DasGupta. Probability for statistics and machine learning. Springer Texts in Statistics. Springer, New York, 2011. Fundamentals and advanced topics.

[11] A. Durmus and E. Moulines. Sampling from strongly log-concave distributions with the Unadjusted Langevin Algorithm. ArXiv e-prints, May 2016.

[12] A. Eberle, A. Guillin, and R. Zimmer. Quantitative Harris type theorems for diffusions and McKean-Vlasov processes. ArXiv e-prints, June 2016.

[13] Andreas Eberle. Reflection coupling and Wasserstein contractivity without convexity. C. R. Math. Acad. Sci. Paris, 349(19-20):1101-1104, 2011.

[14] Andreas Eberle. Reflection couplings and contraction rates for diffusions. Probability Theory and Related Fields, pages 1-36. Published online (DOI) 10.1007/s00440-015-0673-1, 2015.

[15] Hans-Jürgen Engelbert and Goran Peskir. Stochastic differential equations for sticky Brownian motion. Stochastics, 86(6):993-1021, 2014.

[16] Stewart N. Ethier and Thomas G. Kurtz. Markov processes. Wiley Series in Probability and Mathematical Statistics: Probability and Mathematical Statistics. John Wiley \& Sons, Inc., New York, 1986. Characterization and convergence.

[17] William Feller. Diffusion processes in one dimension. Trans. Amer. Math. Soc., 77:1-31, 1954.

[18] William Feller. The general diffusion operator and positivity preserving semi-groups in one dimension. Ann. of Math. (2), 60:417-436, 1954.

[19] Carl Graham. The martingale problem with sticky reflection conditions, and a system of particles interacting at the boundary. Ann. Inst. H. Poincaré Probab. Statist., 24(1):45-72, 1988.

[20] M. Grothaus and R. Voßhall. Construction and analysis of sticky reflected diffusions. ArXiv e-prints, December 2014.

[21] M. Grothaus and R. Voßhall. Strong Feller properties and uniqueness of sticky reflected distorted Brownian motion. ArXiv e-prints, October 2014.

[22] H. Hajri and M. Arnaudon. Application of stochastic flows to the sticky brownian motion equation. ArXiv e-prints, March 2016.

[23] J. Michael Harrison and Austin J. Lemoine. Sticky Brownian motion as the limit of storage processes. J. Appl. Probab., 18(1):216-226, 1981.

[24] Martina Hofmanová and Jan Seidler. On weak solutions of stochastic differential equations. Stoch. Anal. Appl., 30(1):100-121, 2012.

[25] Martina Hofmanová and Jan Seidler. On weak solutions of stochastic differential equations II. Stoch. Anal. Appl., 31(4):663-670, 2013.

[26] Christopher John Howitt. Stochastic flows and sticky brownian motion. PhD Thesis, University of Warwick, 2007.

[27] Nobuyuki Ikeda. On the construction of two-dimensional diffusion processes satisfying Wentzell's boundary conditions and its application to boundary value problems. Mem. Coll. Sci. Univ. Kyoto Ser. A Math., 33:367-427, 1960/1961.

[28] Nobuyuki Ikeda and Shinzo Watanabe. A comparison theorem for solutions of stochastic differential equations and its applications. Osaka J. Math., 14(3):619-633, 1977.

[29] Nobuyuki Ikeda and Shinzo Watanabe. Stochastic differential equations and diffusion processes, volume 24 of North-Holland Mathematical Library. North-Holland Publishing Co., Amsterdam; Kodansha, Ltd., Tokyo, second edition, 1989.

[30] Jean Jacod and Albert N. Shiryaev. Limit theorems for stochastic processes, volume 288 of Grundlehren der Mathematischen Wissenschaften [Fundamental Principles of Mathematical Sciences]. Springer-Verlag, Berlin, second edition, 2003. 
[31] Yu. M. Kabanov, R. Sh. Liptser, and A. N. Shiryaev. On the variation distance for probability measures defined on a filtered space. Probab. Theory Relat. Fields, 71(1):19-35, 1986.

[32] Ioannis Karatzas and Steven E. Shreve. Brownian motion and stochastic calculus, volume 113 of Graduate Texts in Mathematics. Springer-Verlag, New York, second edition, 1991.

[33] Achim Klenke. Probability theory. Universitext. Springer, London, second edition, 2014. A comprehensive course.

[34] F. Liese. Hellinger integrals of diffusion processes. Statistics, 17(1):63-78, 1986.

[35] F. Liese and W. Schmidt. On the strong convergence, contiguity and entire separation of diffusion processes. Stochastics Stochastics Rep., 50(3-4):185-203, 1994.

[36] Torgny Lindvall and L. C. G. Rogers. Coupling of multidimensional diffusions by reflection. Ann. Probab., 14(3):860-872, 1986.

[37] Petr Mandl. Analytical treatment of one-dimensional Markov processes. Die Grundlehren der mathematischen Wissenschaften, Band 151. Academia Publishing House of the Czechoslovak Academy of Sciences, Prague; Springer-Verlag New York Inc., New York, 1968.

[38] O. Manita. Estimates for Kantorovich functionals between solutions to Fokker - Planck Kolmogorov equations with dissipative drifts. ArXiv e-prints, July 2015.

[39] H. P. McKean, Jr. A. Skorohod's stochastic integral equation for a reflecting barrier diffusion. J. Math. Kyoto Univ., 3:85-88, 1963.

[40] Sylvie Méléard. Asymptotic behaviour of some interacting particle systems; McKean-Vlasov and Boltzmann models. In Probabilistic models for nonlinear partial differential equations (Montecatini Terme, 1995), volume 1627 of Lecture Notes in Math., pages 42-95. Springer, Berlin, 1996.

[41] Goran Peskir. On boundary behaviour of one-dimensional diffusions: From brown to feller and beyond. In William Feller - Selected Papers II. Springer, Berlin, 2015.

[42] Enrico Priola and Feng-Yu Wang. Gradient estimates for diffusion semigroups with singular coefficients. J. Funct. Anal., 236(1):244-264, 2006.

[43] Philip E. Protter. Stochastic integration and differential equations, volume 21 of Stochastic Modelling and Applied Probability. Springer-Verlag, Berlin, 2005. Second edition. Version 2.1, Corrected third printing.

[44] Miklós Z. Rácz and Mykhaylo Shkolnikov. Multidimensional sticky Brownian motions as limits of exclusion processes. Ann. Appl. Probab., 25(3):1155-1188, 2015.

[45] Anatoliy V Skorokhod. Stochastic equations for diffusion processes in a bounded region. Theory of Probability $\&$ Its Applications, 6(3):264-274, 1961.

[46] Anatoliy V Skorokhod. Stochastic equations for diffusion processes in a bounded region. ii. Theory of Probability \& Its Applications, 7(1):3-23, 1962.

[47] Daniel W. Stroock and S. R. S. Varadhan. Diffusion processes with boundary conditions. Comm. Pure Appl. Math., 24:147-225, 1971.

[48] Daniel W. Stroock and S. R. Srinivasa Varadhan. Multidimensional diffusion processes. Classics in Mathematics. Springer-Verlag, Berlin, 2006. Reprint of the 1997 edition.

[49] Alain-Sol Sznitman. Topics in propagation of chaos. In École d'Été de Probabilités de SaintFlour XIX-1989, volume 1464 of Lecture Notes in Math., pages 165-251. Springer, Berlin, 1991.

[50] Cédric Villani. Optimal transport, volume 338 of Grundlehren der Mathematischen Wissenschaften [Fundamental Principles of Mathematical Sciences]. Springer-Verlag, Berlin, 2009. Old and new.

[51] Jonathan Warren. Branching processes, the Ray-Knight theorem, and sticky Brownian motion. In Séminaire de Probabilités, XXXI, volume 1655 of Lecture Notes in Math., pages 1-15. Springer, Berlin, 1997.

[52] Shinzo Watanabe. On stochastic differential equations for multi-dimensional diffusion processes with boundary conditions. J. Math. Kyoto Univ., 11:169-180, 1971.

[53] Shinzo Watanabe. On stochastic differential equations for multi-dimensional diffusion processes with boundary conditions. II. J. Math. Kyoto Univ., 11:545-551, 1971. 
[54] R. Zimmer. Explicit contraction rates for a class of degenerate and infinite-dimensional diffusions. ArXiv e-prints, May 2016.

Universität Bonn, Institut für Angewandte Mathematik, Endenicher Allee 60, 53115 Bonn, Germany

E-mail address: eberle@uni-bonn.de

$U R L$ : wt.iam.uni-bonn.de

Universität Bonn, Institut fÜr Angewandte Mathematik, Endenicher Allee 60, 53115 Bonn, GERmany

E-mail address: Raphael.Zimmer@uni-bonn.de

$U R L$ : wt.iam.uni-bonn.de 